\title{
Design of a novel vaccine nanotechnology-based delivery system comprising CpGODN-protein conjugate anchored to liposomes
}

Despo Chatzikleanthous ${ }^{1}$, Signe Tandrup Schmidt ${ }^{1,4}$, Giada Buffi ${ }^{2}$, Ida Paciello ${ }^{2}$, Robert Cunliffe ${ }^{1}$, Filippo Carboni ${ }^{2}$, Maria Rosaria Romano ${ }^{2}$, Derek T. O'Hagan ${ }^{3}$, Ugo D'Oro ${ }^{2}$, Stuart Woods ${ }^{1}$, Craig W. Roberts ${ }^{1}$, Yvonne Perrie ${ }^{1}$, Roberto Adamo ${ }^{2 *}$.

${ }^{1}$ Strathclyde Institute of Pharmacy and Biomedical Sciences, University of Strathclyde, 161 Cathedral St, G4 ORE, Glasgow, UK

${ }^{2}$ GSK, Via Fiorentina 1, 53100, Siena, Italy

${ }^{3}$ GSK, 14200 Shady Grove Rd, Rockville, MD

${ }^{4}$ Department of Infectious Disease Immunology, Center for Vaccine Research, Statens Serum Institut, Artillerivej 5, 2300 Copenhagen S, Denmark

Key words: TLR9 agonist; Cationic Liposomes; Nanoparticles; Conjugation; Vaccines; Group B Streptococcus

Corresponding author contact information:

Dr. Roberto Adamo

Research Centre, Bld 35

GSK

Via Fiorentina 1, 53100, Siena

E-mail: roberto.x.adamo@gsk.com 
Although the well-known Toll like receptor 9 (TLR9) agonist CpGODN has shown promising results as vaccine adjuvant in preclinical and clinical studies, its in vivo stability and potential systemic toxicity remain a concern. In an effort to circumvent these issues, different strategies have been employed to increase its stability, localise action and reduce dosage. These include conjugation of CpGODN with proteins or encapsulation/adsorption of CPGODN into/onto liposomes and have resulted in enhanced immunopotency compared to co-administration of free CpGODN and antigen. Here, we designed a novel delivery system of CpGODN based on its conjugation to serve as anchor for liposomes. Thiolmaleimide chemistry was utilised to covalently ligate the Group B Streptococcus (GBS) GBS67 protein antigen with the CPGODN TLR9 agonist. This treatment did not alter protein's ability to be recognised by specific antibodies or the CpGODN to function as a TLR9 agonist. Due to its negative charge, the protein conjugate readily electrostatically bound cationic liposomes composed of 1, 2-distearoyl-snglycero-3-phosphocholine (DSPC), cholesterol and dimethyldioctadecylammonium bromide (DDA). The novel cationic liposomes-protein conjugate complex (GBS67-CpGODN+L) shared similar vesicle characteristics (size and charge) compared to free liposomes but exhibited different structure and morphology. Following intramuscular immunisation, GBS67-CpGODN+L formed a vaccine depot at the injection site and induced a remarkable increase of functional immune responses against GBS compared to the simple co-administration of GBS67, CpGODN and liposomes. This work demonstrates that the conjugation of CPGODN to GBS67 in conjunction with adsorption on cationic liposomes, can promote co-delivery leading to the induction of a multifaceted immune response at low antigen and CpGODN doses. Our findings highlight the potential for harnessing the immunostimulatory properties of different adjuvants to develop more effective nanostructure-based vaccine platforms. 


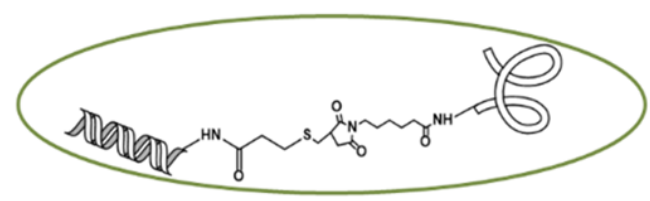

Increase vaccine potency

Surface exposed
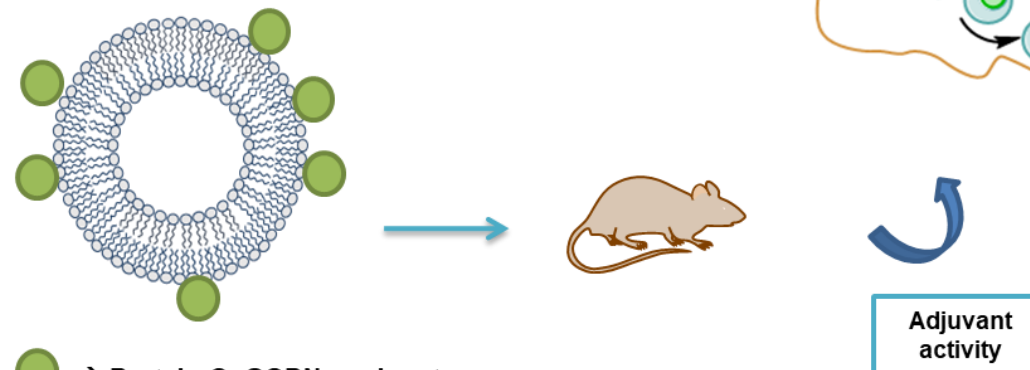

$\rightarrow$ Protein-CpGODN conjugate activity

$\rightarrow$ Cationic liposomes composed by DSPC:Cholesterol:DDA 10:40:50 molar ratio

\section{Introduction}

CpG oligodeoxynucleotides (CpGODN) are short single-stranded synthetic DNA molecules that include of a cytosine triphosphate deoxynucleotide and a guanine triphosphate deoxynucleotide. They mimic microbial DNA that often contains these motifs and their ability to enhance immune responses is well documented $[1,2]$. Responsiveness to CpG motifs is mediated through TLR9, a receptor localised to and signalling from the endosomal compartment of antigen presenting cells (APCs), such as dendritic cells (DCs) and macrophages. TLR9 binding of CpG-containing DNA results in the induction of rapid innate immune responses to prevent or limit early infection, but crucially also directs the quality of the specific adaptive immune response to facilitate pathogen clearance and, finally, memory responses for long-lived protection. Supported by the induction of immunostimulatory T helper Th1biasing cytokines and chemokines including interleukin IL-12, tumor necrosis factor TNF- $\alpha$ and interferon IFN $\alpha / \beta$ and $\gamma$, CpGs directly (i.e., APCs) or indirectly (i.e., natural killer cells and T lymphocytes) activate a variety of immune cells, ultimately resulting in enhanced immune function [3].

CpGODN have shown promising results in preclinical and clinical studies, and recently Heplisav B, a prophylactic vaccine combining the Hepatitis B surface antigen HBsAg adjuvanted with CpG1018 ISS, has been licensed [4]. Despite this success, the use of CpGODN is associated with several obstacles including poor in vivo stability mainly due to their digestion by endonucleases, unfavourable 
pharmacokinetic and biodistribution profiles and poor cellular uptake characteristics [1]. In addition, there are safety concerns regarding undesirable side effects observed depending on the administered dose [5]. These side effects include liver toxicity, enlargement of the lymph nodes [6], extramedullary hematopoiesis [7], systemic inflammation [8-10] and renal damage [11]. Additionally, autoimmune responses have been observed in cancer patients [12]. Reduction in such effects could be achieved by lowering the dose of administered compound.

In an effort to overcome these issues, alternative in vivo delivery systems of CpGODN, including conjugation strategies and nanoparticulate formulations, have been suggested. Conjugation of CpG motifs with protein antigens creates a more potent immunogen compared to physical mixture of antigen and CpG [13]. Co-localisation, improved antigen uptake and presentation, and thus enhanced immune responses are some of the benefits of such protein conjugates. Specifically, whilst proteinCpG mixtures have the limitation of inconsistent co-localisation, protein-CpG conjugates ensure efficient internalisation of antigen and adjuvant by the same DCs through endocytosis and activation of the intracellular TLR9, allowing the use of lower doses of adjuvant compared to the unconjugated form [14-16].

As an alternative to conjugation, liposomal delivery of CpGODN has been demonstrated to offer important advantages including protection from DNase degradation, extension of retention time inside the body, improved cellular uptake, delivery to target tissues and slow release over a long period of time [17]. Various types of liposomal CpGODN have been developed to achieve immunostimulation, and encapsulation or co-administration of $\mathrm{CpG}$ motifs into/with liposomes have been shown to dramatically enhance the potency of immunogens compared to free CpGODN [18-21].

In this context, we explored the potential of protein-CpGODN conjugate anchored to liposome nanoparticles by adsorption to enhance immunogenicity. It was anticipated that the covalent linkage of the TLR9 agonist CpGODN to a protein antigen multivalently presented on the surface of cationic liposomes could promote accumulation of protein and adjuvant within the body, facilitate their delivery and further increase vaccine efficiency compared to protein conjugation alone or liposome delivery.

To test this hypothesis, we addressed our attention to the pathogenic bacteria Streptococcus agalactiae or Group B Streptococcus (GBS) [22-25]. GBS are gram-positive, $\beta$-haemolytic, chainforming cocci that are normal residents of the vaginal flora in $25 \%$ of healthy women [26]. GBS can convert from the asymptomatic mucosal carriage state to a bacterial pathogen causing infections in pregnant women and newborns. The main transmission of GBS is maternal transmission leading to serious neonatal infections including meningitis, sepsis and pneumonia. GBS is a leading cause of 
neonatal mortality and morbidity globally [27]. It is estimated that out of 410,000 cases every year, there will be at least 147,000 stillbirths and infant deaths worldwide [28] and permanent neurologic sequelae in the majority of survivors [29]. A vaccine against GBS is not available yet, and antibiotic resistance is becoming a concern [30-32]. Pilus proteins have been identified through reverse vaccinology as promising vaccine candidates, as they play a key role in the adhesion and attachment of gram-negative and gram-positive pathogens to host cells [33]. Three pilus variants (PI-1, PI-2a and $\mathrm{PI}-2 \mathrm{~b}$ ) are present in the human GBS pathogen, and for this study we selected as model GBS67, an ancillary highly conserved three-domain protein of PI-2a variant.

\section{Materials and methods}

\subsection{Materials}

CpGODN 1826 (5'-[AmC6]TCCATGACGTTCCTGACGTT), N-e-malemidocaproyl-oxysuccinimide ester (EMCS), succinimidyl 3-(2-pyridyldithio)propionate (SPDP), Tris(2-carboxyethyl)phosphine hydrochloride solution (TCEP), sinapinic acid, peroxidase-labelled goat anti-mouse IgG, IgG1, IgG2a were purchased from Sigma-Aldrich (Poole, Dorset, UK). Cholesterol, 1,2-distearoyl-sn-glycero-3phosphocholine (DSPC), Dimethyldioctadecylammonium (DDA) were purchased from Avanti Polar Lipids (Alabaster, AL, USA). GBS67 was supplied by GSK (Siena, Italy). Alexa Fluor 790 protein labelling kit and 1,1'-Dioctadecyl-3,3,3',3'-Tetramethylindocarbocyanine Perchlorate (DiD) lipophilic tracker were obtained from ThermoFisher Scientific (Loughborough, UK).

\subsection{Chemical synthesis}

\subsubsection{Preparation of GBS67-EMCS}

GBS67 was modified as previously described [34]. Briefly, $1.52 \mathrm{mg}$ of EMCS were dissolved in $50 \mu \mathrm{L}$ of DMSO, and $11 \mu \mathrm{L}$ of the prepared mixture (6 eq-v) was added to a solution of GBS67 (327 $\mu \mathrm{L}$ of 18.4 $\mathrm{mg} / \mathrm{mL}$ stock solution) in $670 \mu \mathrm{L}$ of $100 \mathrm{mM} \mathrm{NaPi} 1 \mathrm{mM}$ EDTA pH 8.1. Reaction was incubated for 3 hours at RT. After 3 hours, GBS67-EMCS was purified using $30 \mathrm{kDa}$ Vivaspin filter unit $0.5 \mathrm{~mL}$ (x5 cycles) dialysing against $50 \mathrm{mM} \mathrm{NaPi}, 1 \mathrm{mM}$ EDTA pH 7.5. Protein content was determined by BCA colorimetric assay. The linker/protein molar ratio was determined by MALDI-TOF mass spectrometry analysis run in an UltraFlex III MALDI-TOF/TOF instrument (Bruker Daltonics) in linear mode and with positive ion detection. The samples for analysis were prepared by mixing $2.5 \mu \mathrm{L}$ of product and $2.5 \mu \mathrm{L}$ of sinapinic acid matrix. $2.5 \mu \mathrm{L}$ of mixture was deposited on a samples plate, dried at RTfor $10 \mathrm{~min}$, and subjected to the spectrometer.

\subsubsection{Preparation of CpGODN-SH}

An amount of $20 \mathrm{mg}(3.21 \mu \mathrm{mol})$ of CpGODN 1826 (5'-[AmC6] TCCATGACGTTCCTGACGTT, MW 6238) were reacted with 10 eq-v $(10 \mathrm{mg}, 32.1 \mu \mathrm{mol})$ of SPDP linker in 1:9 (v/v) $100 \mathrm{mM} \mathrm{NaPi} \mathrm{pH} \mathrm{7.2:} \mathrm{DMSO}$ 
137 (1 mL). The reaction was incubated for 3 hours at RT under continuous mixing and was purified by size exclusion chromatography on a G25 Sephadex column eluting with $\mathrm{H}_{2} \mathrm{O}$. Fractions contained the CpGODN-SH were combined and concentrated. ${ }^{1} \mathrm{H}$ NMR was performed in order to assess the success of the reaction (Fig. S1 Supporting material). To release the free thiol groups, CpGODN-SH was treated with 3 eq-v of 0.0005 M TCEP solution for 3 hours at RT in the dark. The reaction mixture was purified by size exclusion chromatography using G25 Sephadex column and $\mathrm{H}_{2} \mathrm{O}$ as eluent. The amount of CPGODN-SPDP recovered was quantified by measuring UV absorbance at $260 \mathrm{~nm}$.

\subsubsection{Conjugation of CpGODN-SH to GBS67-EMCS}

Protein conjugate was prepared by incubating GBS67-EMCS with CpGODN-SPDP (1:10 eq protein: CpGODN) in $50 \mathrm{mM} \mathrm{NaPi}, 1 \mathrm{mM}$ EDTA pH 7.5. The reaction was incubated overnight at RT under continuous mixing. Protein conjugate was purified using $30 \mathrm{kDa}$ Vivaspin filter unit $0.5 \mathrm{~mL}$ ( $x 40$ dialysis cycles) and recovered in PBS (1x) buffer. The protein and CpGODN content were determined by BCA colorimetric assay and UV absorbance at $260 \mathrm{~nm}$, respectively. Finally, the extent of protein conjugation to CpGODN was evaluated by SDS-PAGE electrophoresis and SEC-HPLC.

\subsection{NF-kB luciferase reporter assay}

TLR-specific activation assay was performed using human embryonic kidney 293 (HEK293) cells expressing luciferase under control of the NF-KB promoter and stably transfected with mice TLR9. HEK293-transfected cells were maintained in DMEM complemented with $4.5 \mathrm{~g} / \mathrm{L}$ glucose and HEPES (Invitrogen), 10\% fetal bovine serum (FBS), 1\% penicillin/streptomycin solution (Invitrogen), puromycin $(5 \mu \mathrm{g} / \mathrm{mL})$ and blasticidin $(5 \mu \mathrm{g} / \mathrm{mL})$. For the NF-kB luciferase assay, 25,000 cells/well were seeded in $90 \mu \mathrm{L}$ of complete DMEM without antibiotics in 96 -well $\mu$ Clear luciferase plates (PBI International) and incubated for 24 hours at $37^{\circ} \mathrm{C}$. Successively, cells were stimulated with $10 \mu \mathrm{L}$ of serial 2-fold dilutions of GBS67-CpGODN or CpGODN alone in PBS (Starting concentration $50 \mathrm{\mu g} / \mathrm{mL}$ ). All compounds concentrations were tested in triplicate. After incubation for 6 hours at $37^{\circ} \mathrm{C}$, supernatants were discarded from each well, and cells were lysed for 20 minutes at RT using 20 $\mu \mathrm{L} /$ well of 1:5 diluted 'passive lysis buffer'(Promega). Luciferase assay reagent (100 $\mu \mathrm{L} /$ well) (Promega) was added, and emitted light was immediately quantified using a microplate reader (Tecan, Männedorf, Switzerland). NF-KB activation of stimulated cells was expressed as fold-increase in emitted light over the average result of PBS stimulated control cells.

\subsection{Dot blotting}

167 Test sample and controls $(2 \mathrm{mg} / \mathrm{mL})$ spotted on nitrocellulose membranes and the membranes were allowed to dry for 20 minutes. Non-specific sites were blocked by soaking the membranes in PBS-5\% w/v BSA-0.05\% v/v Tween20 $10 \mathrm{~cm}$ Petri dishes for 1 hour at RT. After 3 washes with PBS $0.05 \% \mathrm{v} / \mathrm{v}$ 
Tween20, membrane was incubated with either anti-GBS67 serum diluted in 1:1000 with PBS-5\% w/v BSA-0.05\% v/v Tween20 (positive sera) or serum from non-immunised mice (1:1000 in PBS-5\% w/v BSA-0.05\% v/v Tween20) (negative sera) for 1 hour at RT. Membranes were washed three times with PBS $0.05 \% \mathrm{v} / \mathrm{v}$ Tween20 ( $3 \times 5$ minutes). Finally, membranes were incubated with fluorescent antimice IgG secondary antibody (1:10000 in PBS-5\% w/v BSA-0.05\% v/v Tween20) for 45 minutes at RT. After final washing with PBS $0.05 \% \mathrm{v} / \mathrm{v}$ Tween20 ( $3 \times 5$ minutes), membranes were scanned using Odyssey fluorescent imaging system (LI-COR Biosciences, Lincoln, NE, USA).

\subsection{Preparation of liposomes}

178 The preparation of DSPC: Cholesterol: DDA cationic liposomes was achieved via microfluidics 179 (Nanoassemblr, Precision NanoSystems Inc., Vancouver, Canada) processes based on previously 180 developed methods (e.g. [35-37]. Briefly, DSPC: Cholesterol: DDA lipid mixture was prepared in ethanol at 10:40:50 molar ratio. Then, the lipids and an aqueous phase (10 mM TRIS buffer pH 7.4) were injected simultaneously in the micromixer. All formulations were prepared at $20 \mathrm{mg} / \mathrm{mL}$ initial lipid concentration, 1:1 aqueous: organic flow rate ratio (FRR) and $12 \mathrm{~mL} / \mathrm{min}$ total flow rate (TFR). All newly formed liposomes $(1 \mathrm{~mL})$ were then subjected to buffer exchange via dialysis against $10 \mathrm{mM}$ TRIS pH 7.4 for 1 hour under magnetic stirring to ensure removal of solvent residual. For biodistribution studies, liposomes were formulated incorporating the lipophilic fluorescent dye DiD (1:100 w/v DiD: Total lipid) within the bilayer. Any non-incorporated free DiD dye (MW = $933 \mathrm{Da}$ ) was removed via dialysis (14 kDa MWCO).

To investigate the adsorption of protein- $\mathrm{CpG}$ conjugate onto liposomes surface, GBS67-CpGODN was mixed with DSPC: Cholesterol: DDA liposomes to a similar manner as reported before [38]. Briefly, liposomes were incubated with GBS67-CpGODN (1:50 w/w protein: liposomes) in 10mM TRIS pH 7.4. To serve as controls, liposomes adsorbed free GBS67 or free GBS67 and CpGODN were also prepared. Samples were left to equilibrate for 30 minutes in RT. Dialysis using Biotech CE tubing ( $300 \mathrm{kDa}$ MWCO) was carried out overnight at $4^{\circ} \mathrm{C}$ with two buffer changes, for removal of unbound protein. BCA assay and UV were used for quantification of protein $(280 \mathrm{~nm})$ and CpGODN (260 nm), respectively. The amount of protein adsorbed on liposomes surface was calculated by subtracting the amount of protein remaining in solution from the amount of protein initially added to the liposome dispersion.

\subsection{Liposome characterisation}

The size distribution (mean diameter and polydispersity index (PDI)) and the zeta potential of the liposomes were measured by dynamic light scattering using photon correlation spectroscopy on a 
being diluted in 1/10 using their aqueous phase (1:300 v/v $10 \mathrm{mM}$ TRIS pH 7.4). Sizes quoted are the $\mathrm{z}$-average mean for the liposomal hydrodynamic diameter $(\mathrm{nm})$.

\subsection{Liposome morphology}

Geometry of liposomes was observed by cryo-TEM as described previously by others [39-41] with minor modifications. For TEM observations, a Jeol Jem F-200 microscope (Jeol, Tokyo, Japan) operating at $200,000 \mathrm{~V}$ was used. Samples were prepared by placing $5 \mu \mathrm{L}$ of liposomes onto a 400 mesh lacey carbon-coated grid, blotting from both sides for approximately $2 \mathrm{~s}$ and then plunging into nitrogen cooled ethane ( $100 \%$ ethane). Samples were then observed in a cryo-holder in electron microscope Jem F-200 microscope (Jeol, Tokyo, Japan) at liquid nitrogen temperature and $200 \mathrm{KV}$.

\subsection{Fluorolabelling of GBS67 protein and GBS67-CpGODN protein conjugate}

GBS67 protein and GBS67-CpGODN protein conjugate were labelled using Alexa Fluor 790 protein labelling kit (Molecular probes) according to the manufacturer's instructions.

\subsection{Biodistribution studies}

All experiments were undertaken in accordance with the regulations of the Directive 2010/63/EU. Female BALB/c mice, 7-12 weeks old were split into 3 groups of 3 mice. All mice were immunised intramuscularly ( $50 \mu \mathrm{L} /$ dose) at day 0 with fluorolabeled antigens (Alexa Fluor 790 ) and liposomes (DiD). Mice anesthetised with isoflurane were placed into the IVIS chamber, and images were captured using the IVIS spectrum camera (Perkin Elmer) at day 0-4 and then every two days until day 11. Mice were terminated at day14. A non-immunised mouse was used as negative control and for quantifying the background level. The final antigen, CpGODN and liposomes concentrations in formulations were $0.2 \mathrm{mg} / \mathrm{mL}, 0.03 \mathrm{mg} / \mathrm{mL}$ and $4 \mathrm{mg} / \mathrm{mL}$, respectively.

\subsection{Immunisation studies}

All experiments were undertaken in accordance with the regulations of the Directive 2010/63/EU. Female BALB/c mice, 6-8 weeks old were split into 6 groups of 5 mice. All mice were immunised intramuscularly ( $50 \mu \mathrm{L} /$ dose) two times (days 0 and 21 ) and at scheduled time points, blood samples were taken from the tail $($ day 0,21$)$ and stored at $-20^{\circ} \mathrm{C}$ for future analysis of antibodies. Mice were terminated at day 42 and further processed for isolation of splenocytes. Prior to immunisation, endotoxin levels for the conjugates were tested and confirmed to be below $20 \mathrm{EU} / \mathrm{mL}$ in the final formulations. 
Direct enzyme-linked immunosorbent assay titres of protein antibodies were determined using the coating reagent GBS67. Microtiter plates (Nunc Maxisorp) were coated by adding $100 \mu \mathrm{L}$ per well of coating reagent $(2 \mu \mathrm{g} / \mathrm{mL})$ in PBS pH 7.4. The plates were incubated overnight at $4^{\circ} \mathrm{C}$ and were washed with PBS containing $0.05 \% \mathrm{v} / \mathrm{v}$ Tween 20 and then blocked with $2 \% \mathrm{w} / \mathrm{v}$ BSA in PBS for 1 hour at $37^{\circ} \mathrm{C}$. The wells were then filled with $100 \mu \mathrm{L}$ of serum serially diluted in PBS and incubated at $37^{\circ} \mathrm{C}$ for 2 hours. After 3 washes, $100 \mu \mathrm{L}$ per well of peroxidase-labelled goat anti-mouse (IgG 1:1000, IgG1 1:20,000, IgG2a 1:1000; Sigma-Aldrich) was added and plates incubated for 1 hour at $37^{\circ} \mathrm{C}$. The plates were again washed 3 times with PBS containing $0.05 \% \mathrm{v} / \mathrm{v}$ Tween 20, and finally $100 \mu \mathrm{L}$ of peroxidase substrate solution (Sigma-Aldrich) was added to each well, following incubation of the plates for 30 minutes at RT. The reaction was stopped by the addition of $100 \mu \mathrm{L}$ of $0.2 \mathrm{M} \mathrm{H}_{2} \mathrm{SO}_{4}$ and the plates were read immediately at $450 \mathrm{~nm}$.

\subsection{Isolation and stimulation of splenocytes}

Mice spleens were removed aseptically and placed into universals containing $5 \mathrm{~mL}$ of complete media (RPMI 1640 containing 10\% v/v FCS, 1\% v/v Pen-Strep and 1\% v/v L-glutamine) and kept ice-cold until ready to proceed. Cell suspensions were prepared by forcing spleens through cell strainers with the plunger of a $2 \mathrm{~mL}$ syringe. Cells were transferred to a $50 \mathrm{~mL}$ centrifuge tubes and cell strainers were washed with another $5 \mathrm{~mL}$ of media, respectively. After centrifugation $\left(1200 \mathrm{rpm}, 4^{\circ} \mathrm{C}\right)$ for 5 minutes, cell pellets were resuspended in $3 \mathrm{~mL}$ Boyle's solution (1:9 v/v 0.17 M TRIS: $0.16 \mathrm{M}$ ammonium chloride) and centrifuged at 1200 rpm for 5 minutes for erythrocytes removal. Pellets are then washed twice in $5 \mathrm{~mL}$ complete medium and centrifuged at $1200 \mathrm{rpm}$ for 5 minutes. After final wash, pellets were resuspended in $5 \mathrm{~mL}$ complete media. Viable cell numbers were estimated by trypan blue exclusion. After counting, cells were diluted in complete RPMI so that there were $5 \times 10^{6}$ cells $/ \mathrm{mL}$. A volume of $100 \mu \mathrm{L}$ of cells were added to the appropriate wells of Nunclon 96-well round bottom plate. The same procedure was followed for splenocytes coming from the rest of the immunisation groups. Cells were stimulated with either RPMI media as a negative control or GBS67 antigen ( $4 \mu \mathrm{g} / \mathrm{mL})$ as the investigated antigen. Splenocytes were incubated at $37^{\circ} \mathrm{C}, 5 \% \mathrm{CO}_{2}$ for 72 hours. After 3 days, plates were stored at $-20^{\circ} \mathrm{C}$ for cytokine analysis at a later date.

\subsection{Cytokine analysis of stimulated splenocytes}

Cytokine profiles of supernatants from restimulated splenocytes were analysed using LEGENDplex mouse Th cytokine (13-plex) multi-analyte flow assay kit (Biolegend, San Diego, CA, USA) according to the manufacturer's instructions. 
The functional activity of the sera was determined by OPKA as previously described by Nilo et al. [25]. HL60 cells were grown in RPMI 1640 with $10 \%$ fetal calf serum, incubated at $37^{\circ} \mathrm{C}, 5 \% \mathrm{CO}_{2}$. $\mathrm{HL}-60$ cells were differentiated to neutrophils with $0.78 \%$ dimethylformamide (DMF) and after 4-5 days were used as source of phagocytes. The assay was conducted in 96-well microtiter plate, in a total volume of $125 \mu \mathrm{L} /$ well. Each reaction contained heat inactivated test serum $(12.5 \mu \mathrm{L})$, GBS $\left(6 \times 10^{4}\right.$ colony forming units [CFU]), differentiated HL-60 cells $\left(2 \times 10^{6}\right.$ cells) and $10 \%$ baby rabbit complement (Cederlane) in Hank's balanced salt solution red (Gibco). For each serum sample, six serial dilutions were tested. Negative controls lacked effector cells, or contained either negative sera or heat inactivated complement. After reaction assembly, plates were incubated at $37^{\circ} \mathrm{C}$ for 1 hour under shaking. Before (TO) and after (T60) incubation, the mixtures were diluted in sterile water and plated in Trypticase Soy Agar plates with 5\% sheep blood (Becton Dickinson) . Each plate was then incubated overnight at $37^{\circ} \mathrm{C}$ with $5 \%$ of $\mathrm{CO}_{2}$ counting CFUs the next day. OPA titre was expressed as the reciprocal serum dilution leading to $50 \%$ killing of bacteria and the $\%$ of killing is calculated as follows:

$$
\% \text { killing }=\frac{T_{0-} T_{60}}{T_{0}}
$$

where $T_{0}$ is the mean of the CFU counted at $T_{0}$ and $T_{60}$ is the average of the CFU counted at $T_{60}$ for the two replicates of each serum dilution.

\subsection{Statistical analysis}

Statistical significance was determined by ANOVA followed by Tukey's (HSD) test. Significance was acknowledged for $p$ values less than 0.05. All calculations were made in Minitab 18.

\section{Results and discussion}

\subsection{Protein-TLR9 agonist conjugate assembly}

The synthesis of the CpGODN-protein system was done based on a similar manner to that reported for preparation of other adjuvant-protein conjugates [15, 34, 42, 43]. Maleimide groups were inserted onto GBS67 by reaction of the protein with commercial EMCS linker. An incorporation of 4 maleimides was found by MALDI-TOF analysis of the modified protein (Fig. 2A) [34, 44]. Thiol groups were introduced onto CPGODN 1826 by reaction of the primary amine at $5^{\prime}$ position of the adjuvant molecule with the active ester of SPDP linker. ${ }^{1} \mathrm{H}$ NMR analysis confirmed the successful modification of CpGODN (Fig. S1 Supporting material). After removal of the thio-pyridine protection with TCEP, CpGODN bearing the sulfhydryl groups was incubated with GBS67-EMCS to give addition to the maleimides exposed onto the protein surface (Fig. 1). 


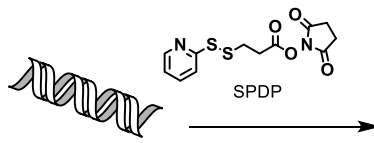

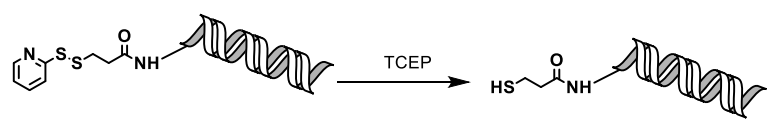
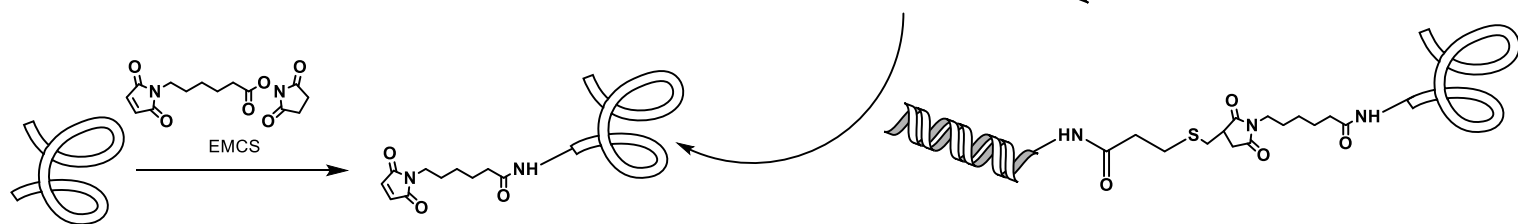

Fig. 1. Reaction scheme for conjugation of CpGODN on GBS67 protein.

SDS-PAGE electrophoresis and SEC-HPLC clearly showed conjugation of the CpGODN to the modified GBS67 antigen (Fig. 2B and C). An average ratio of 4 CpGODN chains was incorporated for each GBS67 molecule in the final product which is in agreement with what has been previously published for the preparation of CPGODN conjugates using other proteins $[15,45,46]$. The characteristics of the protein conjugate are summarised in Table 1.

A

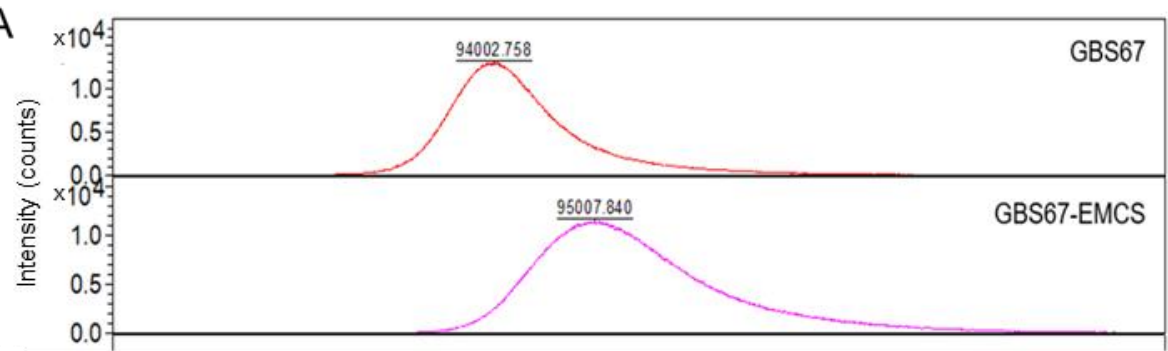

C

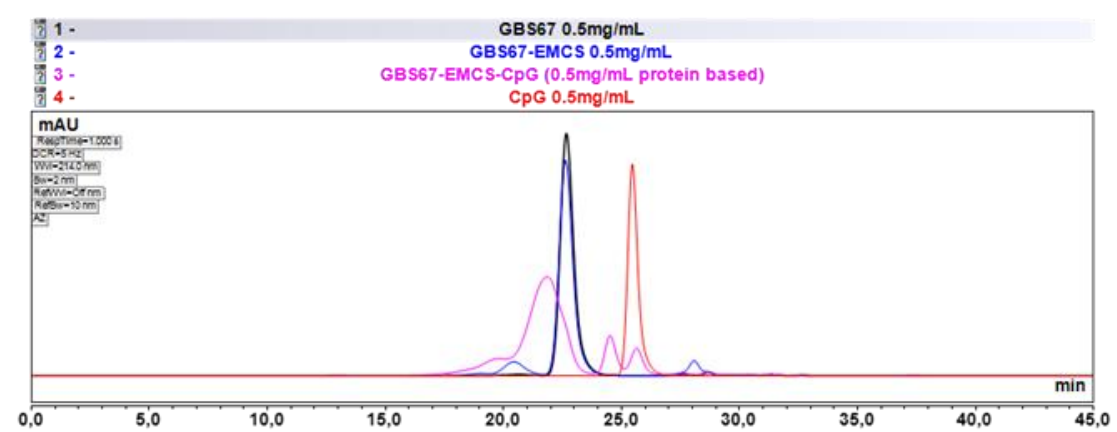

B

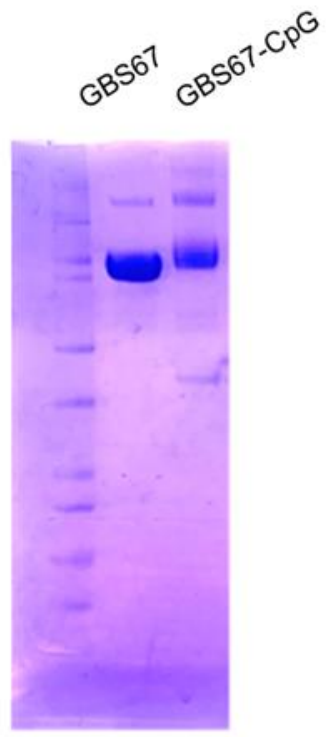

Fig. 2. Characterisation of the formed GBS67-EMCS intermidiate and GBS67-EMCS-SPDP-CpGODN conjugate. (A) MALDI TOF MS analysis of incorporation of EMCS (MW of EMCS $=308.29 \mathrm{Da}$ ) on GBS67, prepared as reported in Ref. [34] (B) SDS-PAGE electrophoresis of native protein in comparison with final conjugate and (C) SEC-HPLC of native GBS67 protein, CpGODN and final conjugate in comparison with GBS67-EMCS intermidiate. 
Table 1. Characteristics of the synthesised conjugate

\begin{tabular}{ccccc}
\hline Conjugate & $\begin{array}{c}\text { CpGODN: protein } \\
\text { stoichiometry } \\
\text { (mol/mol) }\end{array}$ & $\begin{array}{c}\text { MW protein- } \\
\text { CpGODN } \\
\text { conjugate }\end{array}$ & $\begin{array}{c}\text { CpGODN: protein in } \\
\text { conjugate (mol/mol) }\end{array}$ & $\begin{array}{c}{ }^{\text {a Conjugation }} \\
\text { efficiency (\%) }\end{array}$ \\
\hline GBS67-CpGODN & $10: 1$ & 120,000 & $4: 1$ & $40 \%$ \\
\hline
\end{tabular}

310

${ }^{a}$ Amount of conjugated CpGODN vs amount of CpGODN used for conjugation.

\subsection{GBS67-CpGODN is able to activate TLR9}

The capacity of the adjuvanted GBS67 protein to engage TLR9 was evaluated in vitro, using human embryonic kidney 293 (HEK293) cells expressing luciferase under control of the NF-KB promoter and stably transfected with mice TLR9 (Fig. 3A). In this assay, NF-KB activation is measured by monitoring the levels of luciferase expression following stimulation of cells with serial dilutions of TLR9 agonists. The CpGODN 1826 and commercially available CpGODN, were the positive controls. As expected, the conjugated TLR9 showed activation of TLR9 receptor. However, the receptor activation was reduced compared to the positive controls, presumably because the presence of GBS67 was partially hindering and preventing the TLR9 binding to the receptor. We reasoned that combination with cationic liposomes would cause deposition of the adjuvant at the site of injection [47]. Furthermore in vivo internalisation and processing of the protein in APCs could increase the interactions with TLR9 [48].

A

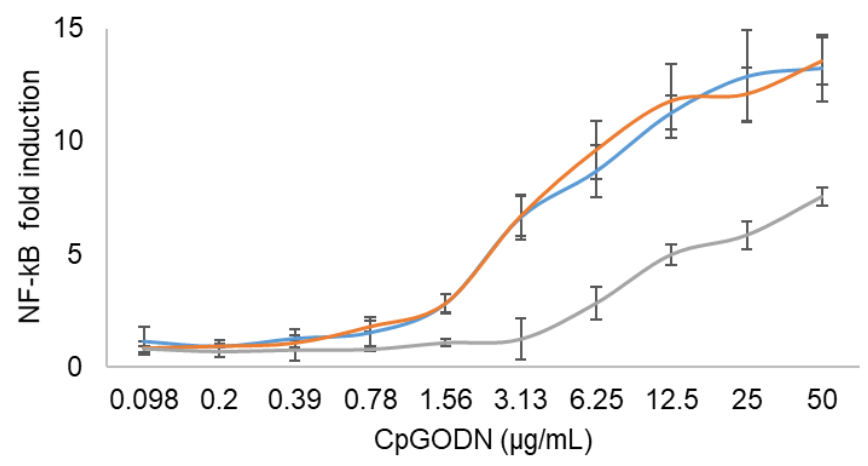

CpGODN CpGODN commercial — GBS67-CpGODN
B

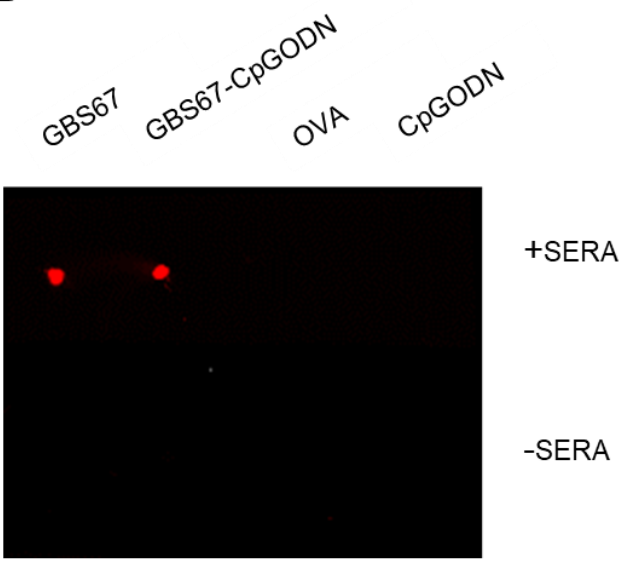

Fig. 3. GBS67-CpGODN conjugate can activate TLR9 and can be recognised by primary anti-GBS67 antibodies. (A) Activation of TLR9 reporter cell line by GBS67-CpGODN conjugate. 25,000 TLR9-HEK293 cells/well were stimulated with $0.1-50 \mu \mathrm{g} / \mathrm{mL}$ (2-fold steps) of TLR9 agonists. Commercial CpGODN as CpGODN used for conjugation were used as a positive controls. After 6 hours, luciferase expression was measured and expressed as fold-induction compared to cells incubated with PBS and plotted as mean \pm SD of duplicates. (B) Dot blotting for GBS67-CpGODN conjugate. Free CpGODN and OVA protein were used as negative controls.

\subsection{GBS67-CpGODN conjugate can be recognised by anti-serum primary antibodies}

The capability of the conjugate to be recognised by primary antibodies was tested using dot blot. The GBS67-CpGODN conjugate was recognised by a murine polyclonal anti-GBS67 serum as well as the unconjugated protein, indicating that the incorporation of CpGODN chains on the protein was not 
impacting on protein epitopes the binding to primary antibodies (Fig. 3B). OVA was used as negative control for the experiment in order to prove antigen specific recognition by the antibodies.

\subsection{Adsorption of GBS67-CpGODN onto cationic liposomes did not induce significant changes in}

DDA-based liposomes have been shown to be effective as delivery systems/adjuvants [47, 49-51] and the aim of this work was to further potentiate the efficacy of these liposomes through the presence of protein-CpGODN on the liposome surface. Accordingly, to consider the impact of the addition of the GBS67-TLR9 agonist conjugate on the formulation, a range of physico-chemical studies were conducted. DLS was used as the entry point measurement for the particle characteristics in order to compare DSPC: Cholesterol: DDA (10:40:50 molar ratio) liposomes with and without GBS67 and CpGODN (GBS67+CpGODN+L) and liposomes prepared with the GBS67-CpGODN conjugate (GBS67CpGODN+L), as also to observe any unexpected failures before the performance of a higher resolution method [52-54]. A formulation with only GBS67 antigen was prepared as control.

The addition of CpGODN $(0.15 \mu \mathrm{g})$ and GBS67 $(1 \mu \mathrm{g})$ resulted in increased particle size of the liposomal formulations without loss of the particle uniformity (PDI between 0.01-0.05; Table 2). Interestingly, while addition of free GBS67 protein or GBS67-CpGODN conjugate on liposomes did not cause a significant change in liposomes size, the highest increase in size (183 $\pm 9 \mathrm{~nm}$; Table 2) was observed when GBS67+CpGODN mixture was used. A reduction in zeta potential was also noted in all the formulations, due the electrostatic interaction of the negatively charged CpGODN and GBS67 with the cationic liposomes, with liposomes having a net surface charge of between +34-39 mV (Table 2).

Table 2. Physicochemical characteristics of liposomal formulations with or without GBS67 and/or CpGODN. Liposomes were mixed with free protein, protein+CpGODN mixture or protein-CpGODN conjugate. The final liposome $(1 \mathrm{mg} / \mathrm{mL})$, protein $(0.02 \mathrm{mg} / \mathrm{mL})$ and CpGODN $(0.003 \mathrm{mg} / \mathrm{mL})$ concentrations in all the samples were equal. Liposomes were characterised in terms of size and PDI by DLS. Results represent the mean \pm SD of two immunisations.

\begin{tabular}{lccc}
\hline & Z-average diameter $(\mathbf{n m})$ & PDI & ZP (mV) \\
\hline Liposomes & $164 \pm 26$ & $0.02 \pm 0.01$ & $45 \pm 5$ \\
GBS67+Liposomes & $166 \pm 26$ & $0.03 \pm 0.02$ & $35 \pm 2$ \\
GBS67-CpGODN+Liposomes & $169 \pm 24$ & $0.05 \pm 0.02$ & $39 \pm 3$ \\
GBS67+CpGODN+Liposomes & $183 \pm 9$ & $0.03 \pm 0.02$ & $34 \pm 1$ \\
\hline
\end{tabular}
formulations tested. The GBS67 antigen and the GBS67-CpGODN conjugate showed pl values of 6.46 and 6.15, respectively, which conferred a net anionic charge, favouring the adsorption to cationic liposomes [38]. To evaluate the association of CpGODN with the liposome formulations, the loading 
association (approximately 100\%), due to the electrostatic interactions between the cationic liposomes and CPGODN. Protein loading was quantified based on a protein:liposome ratio of 0.25:5 $\mathrm{mg} / \mathrm{mL}$ (Fig. S2 Supporting material). Lower protein concentrations were assumed to load to the same efficacy but could not be quantified as the protein concentrations were below the minimum level of detection using the BCA assay. These results are in agreement with what has been previously published by others $[2,55,56]$.

\subsection{Adsorption of GBS67-CpGODN on the surface of cationic liposomes resulted in} morphological changes of liposomes attributes

371 The structure and morphology of the liposomes and GBS67-CpGODN+liposomes complexes was 372 evaluated by cryo-transmission electron microscopy which is one of the most common techniques for 373 nanoparticle size and shape analyse providing the most accurate estimation of the nanoparticle 374 homogeneity [52]. For that purpose, cryo-TEM experiments were run for samples of DSPC: 375 Cholesterol: DDA liposomes and DSPC: Cholesterol: DDA protein conjugate complex at protein ratio 1:50 w/w. Cryo-TEM characterisation of liposomes prior to the addition of the protein conjugate (Fig. $4 \mathrm{~A}$ ) revealed the presence of a homogeneous population of unilamellar spherical liposomes, with an average diameter of around $150 \mathrm{~nm}$ and size distribution similar to sizes measured by DLS (Table 2). Generally, as TEM cannot capture the hydration layer, diameters measured on TEM are often slightly smaller than those obtained by DLS. With the addition of the protein-conjugate, a change in liposome morphology is noted with more elongated or potentially aggregated structures being seen (Fig. 4B, C). This change in morphology was not identified via DLS measurements, which only shows a minor increase in size, with liposomal-conjugates being 180-200 nm (Table 2). Given that DLS is an average particle size based on spherical modelling, it is a less accurate means of sizing such elongated constructs or small populations of aggregates as shown in Figure $4 \mathrm{~B}$ and $\mathrm{C}$. Thus by using cryoTEM we can see that the addition of GBS67-CpGODN does interact with the cationic vesicles resulting in changes in the structure and morphology of the liposomes. 

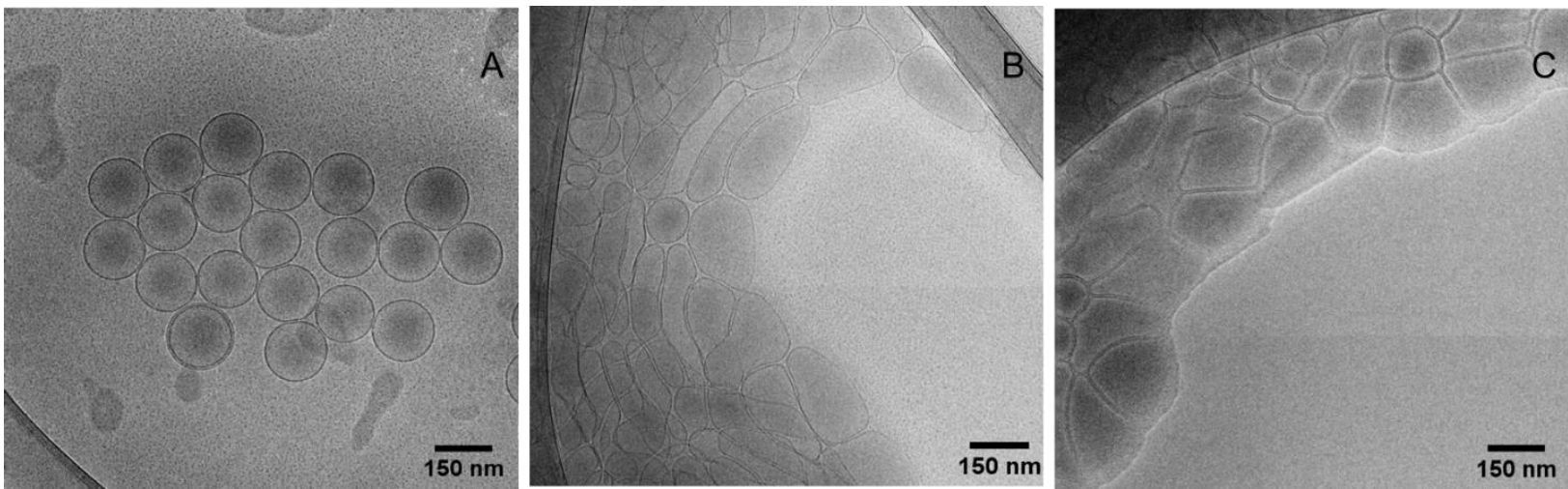

Fig. 4. The effect of GBS67-CpGODN adsorption on liposomes morphology. Cryo-EM images of liposomes (A) before and (B, C) after protein-conjugate adsorption. DSPC: Cholesterol: DDA (10:40:50\% molar ratio) liposome were manufactured using microfluidics at 1:1 FRR, $12 \mathrm{~mL} / \mathrm{min}$ TFR and purified using dialysis. GBS67-CpGODN was mixed with liposomes at 1:50 w/w. The final liposome and GBS67-CpGODN conjugate concentrations in the sample were $5 \mathrm{mg} / \mathrm{mL}$ and $0.1 \mathrm{mg} / \mathrm{mL}$, respectively.

\subsection{Adsorption of GBS67-CpGODN onto cationic liposomes significantly improve antibody}

The induction of humoral responses are of key importance for vaccine efficaciousness [57]. The ability of the designed protein conjugate GSB67-CPGODN to induce functional antibodies was evaluated by immunising groups of $5 \mathrm{BALB} / \mathrm{c}$ mice with two doses of the formulations ( $1 \mu \mathrm{g}$ protein/dose), three weeks apart. The protein dose was selected to better discriminate potential differences produced by the diverse formulations. The novel construct was compared to the physical mixture of GBS67 and CpGODN (GBS67+CpGODN) which contained the same amount of TLR9 agonist and antigen (0.15 $\mu \mathrm{g}$ and $1 \mu \mathrm{g}$, respectively). Both of GBS67 and CpGODN combinations were prepared with and without liposomes (GBS67-CpGODN+L and GBS67+CpGODN+L). Mixture of GBS67 with liposomes (GBS67+L) was used as control to study the effect of the CpGODN incorporation. Pre-immunised mice sera were the negative control.

The anti GBS67 IgG titres after the first dose and boost dose were measured (Fig. 5). Mice immunised with GBS67-CpGODN conjugate showed significantly $(p<0.001)$ higher anti-GBS67 IgG titres compared to non-conjugated physical mixture of GBS67 and CpGODN already after the primary immunisation. The combination of DSPC: Cholesterol: DDA cationic liposomes with either conjugate or physical mixture, further increased the immune responses, with the combination of conjugate and liposomes vaccine giving the highest titre observed after the first injection (Fig. 5A). A similar trend was found after the booster dose, with the GBS67-CpGODN conjugate eliciting the highest IgG titres in the set of tested formulations, significantly $(p<0.001)$ higher than the physical mixture of unconjugated protein and $\mathrm{CpG}$ and the combinations of GBS67 with either CpG or cationic liposomes. Interestingly, CpG gave a poor adjuvant effect on the protein $[14,15]$, due to the low tested dose, while combination of liposomes with GBS67, induced a 4-fold increase of anti-GBS67 titres compared to GBS67 alone. The 
419
A

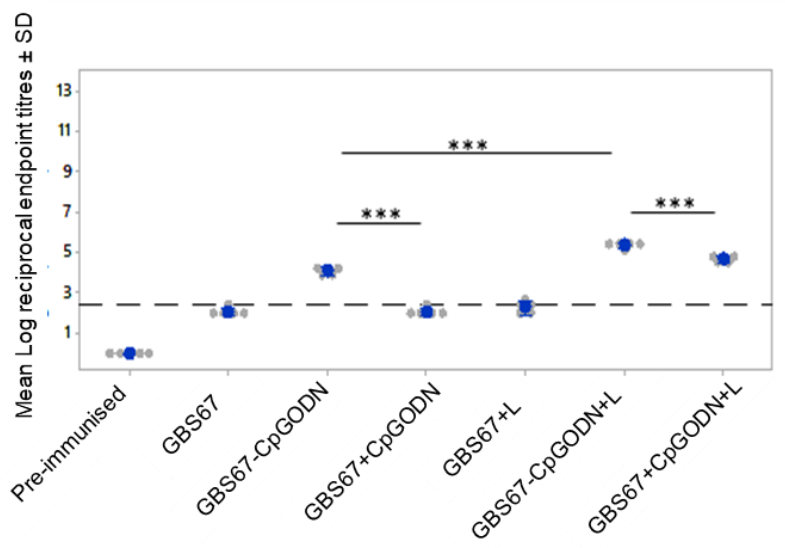

B

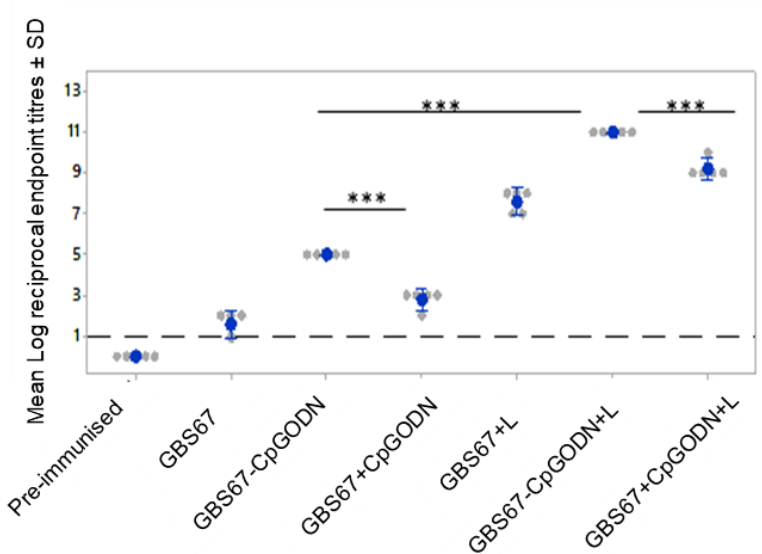

Fig. 5. Total IgG responses after primary dose (A) Day 21 and boost dose (B) Day 42. Six groups of mice were injected twice intramuscularly with the corresponding formulations. The study was split over two experiments with 2 mice from each group in study 1 and 3 mice in study 2. The results are then combined to give an $n=5$. Results are plotted for individual mouse and also an average, so show variability across the studies and mice. Blood samples were taken from the tail at day 21 . Mice were terminated at day 42 and ELISA was performed for determination of total GBS67-specific antibody titre levels. Mixture and conjugate are represented by $(+)$ and $(-)$, respectively. Results are the average of two independent experiments (mean \pm SD). $* * * p<0.001$. Dash line represents the limit of detection.

Since antibody functionality is often linked to specific $\lg G$ subclasses, rather than total lgG levels, the levels of antibody subtypes were analysed by ELISA (Fig. 6). After the primary immunisation, increased levels of IgG1 were observed for GBS67-CpGODN conjugate group which were significantly $(p<0.01)$ higher than free GBS67 and CpGODN physical mixture (Fig. 6A). Although TLR9 agonists are known to polarise towards a Th1 type immune response characterized by increased IgG2a levels, the effect seen here could be explained with the low dose of CpG utilised in this study [2]. The same trend was observed when conjugate and physical mixture of GBS67, CpGODN were combined with liposomes, with GBS67-CpGODN+liposomes giving the higher IgG1 response. Interestingly, no significant differences were obtained between GBS67 and GBS67+CpGODN, as also GBS67+liposomes and GBS67+CpGODN+liposomes, after one dose. Regarding IgG2a, no significant differences were observed between the groups after vaccination with the primary dose (Fig. 6C).

The second dose boosted both IgG1 responses and IgG2a responses (Fig. $6 \mathrm{~B}$ and D, respectively). In particular, the conjugation of TLR9 ligand and/or co-administration with liposomes resulted in an enhanced level of both IgG1 and IgG2a compared to GBS67 alone and GBS67+CpGODN mixture. Following the booster dose, the highest level of IgG isotypes was measured for the group immunised with GBS67-CpGODN conjugate in combination with liposomes. In particular, IgG2a levels were significantly higher than the ones obtained with GBS67+liposomes $(p<0.001)$ and GBS67+CpGODN+liposomes mixture (p<0.01) (Fig. 6D). 

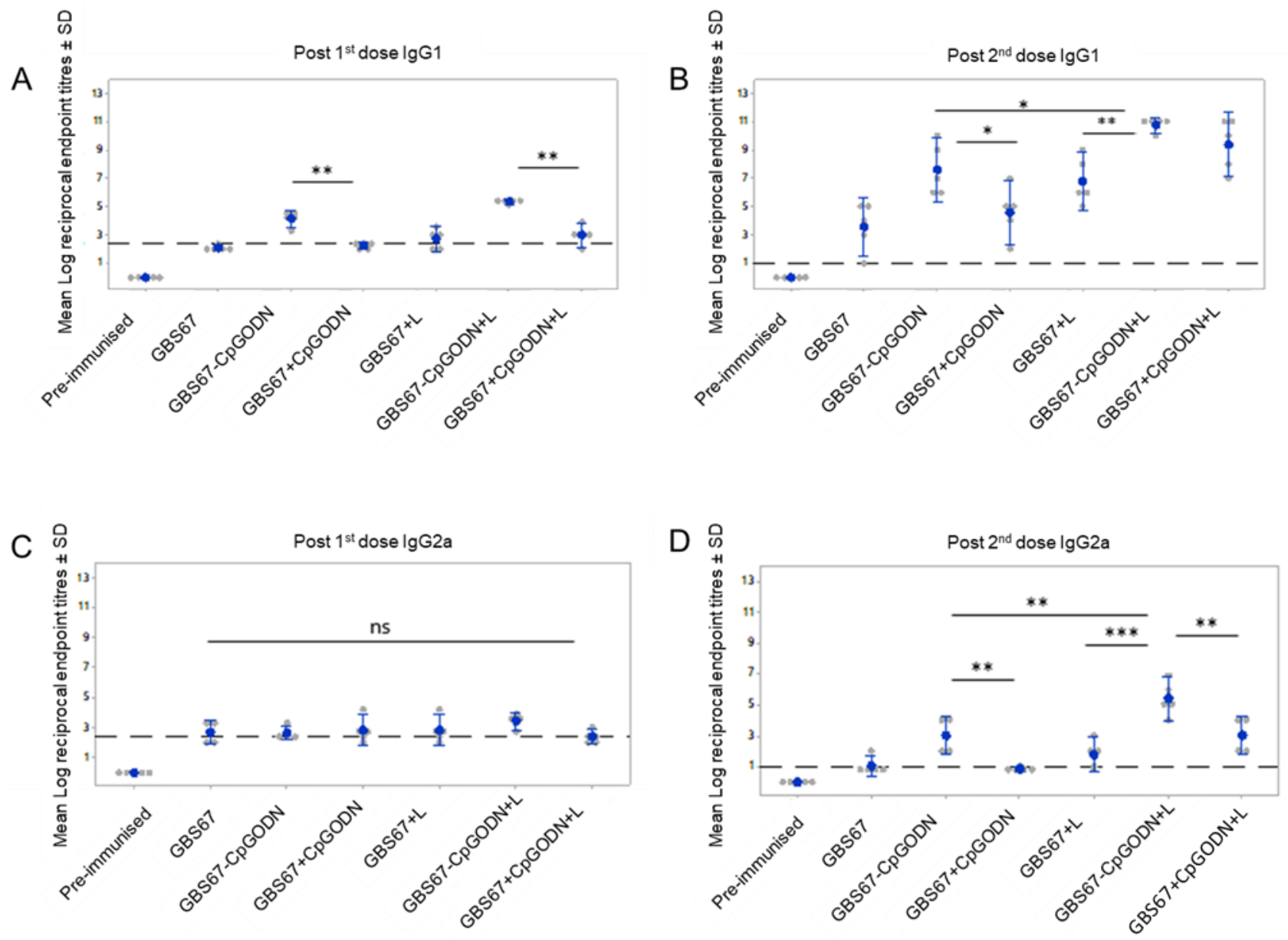

Fig. 6. IgG1 and IgG2a subclasses after primary dose (Day 21, Figure A for IgG1 and C for IgG2a) and boost dose (Day 42 , Figure $B$ for $\operatorname{lgG} 1$ and $D$ for IgG2a). Six groups of mice ( $n=2$ for 1st study 1 and $n=3$ for 2 nd study) were injected twice intramuscularly with the corresponding formulations. Blood samples were taken from the tail at day 21 . Mice were terminated at day 42 and ELISA was performed for determination of Th1 and Th2 antibody-mediated responses. Mixture and conjugate are represented by $(+)$ and $(-)$, respectively. Results are the average of two independent experiments (mean \pm SD). ${ }^{*} p<0.05 ;{ }^{*} p<0.01 ; * * *^{*}<0.001 ;$ ns: non-significant. Dash line represents the limit of detection.

To assess the antibody functionality the pooled sera from vaccinated mice were tested by in vitro killing-based opsonophagocytosis assay (OPKA). This is a well-established assay that mimics in vivo bacterial killing by host effector cells, following opsonisation by specific antibodies and therefore avoiding the need for a challenge study as it is considered a robust surrogate for protection induced by GBS vaccines $[25,58,59]$. A serotype $V$ CJB1111 strain was used to determine effects of the antibodies against GBS67 protein. 
Table 3. Functional activity of designed construct and corresponding controls. OPKA titre was expressed as the reciprocal serum dilution leading to $50 \%$ killing of bacteria. Mixture and conjugate are represented by $(+)$ and $(-)$, respectively. Values represent the mean $\pm \mathrm{SD}$ of three different experiments using pooled sera from each single group ( 5 single mice sera/group). $* * * p<0.001$.

\begin{tabular}{cc}
\hline Group/Serotype V (CJB111) & OPKA titre \pm SD \\
\hline GBS67 & $131 \pm 48$ \\
GBS67-CpGODN & $388 \pm 224$ \\
GBS67+CpGODN & $150 \pm 20$ \\
GBS67+Liposomes & $134 \pm 55^{* *}$ \\
GBS67-CpGODN+Liposomes & $823 \pm 73^{*}$ \\
GBS67+CpGODN+Liposomes & $134 \pm 88^{2}$ \\
\hline
\end{tabular}

468

Adsorption of protein conjugate on the surface of cationic liposomes resulted in a 2-fold and 6-fold increase of functional activity in comparison to GBS67-CpGODN and GBS67, respectively. In contrast, co-administration of liposomes, GBS67 and CpGODN in mixture or combination of GBS67+CpGODN and GBS67+liposomes did not offer any further benefit in terms of serum functional activity, as OPKA titres were comparable to the free GBS67 control. Taking in considerations that 3-fold differences for pooled sera are within the assay variability, GBS67-CpGODN conjugate exhibited an OPKA titre comparable to that of GBS67 control. These suggest that OPKA might be correlated to IgG2a rather than total IgG levels, and at the CpG administered dose, conjugation as also inclusion of liposomes are needed to ensure adjuvant activity(Table 3).

Overall, these results proved that the chemical linkage of TLR9 agonist CPGODN with antigenic protein confers a faster and stronger adjuvant effect towards protein antigen compared to co-administration of antigen and CPGODN, as previously reported [14-16, 45, 46, 60]. The direct linkage of protein antigen and $C p G$, ensures antigen and CpGODN uptake by the same APCs, resulting in a higher response toward the antigen, with a lower adjuvant dose [61-63].

Importantly, liposomal delivery of the GBS67-CpGODN further enhanced the vaccine potency through synergy of multiple display of antigen and TLR9a, and the intrinsic adjuvant effect of liposomes [57].

3.7 Combination of GBS67-CpGODN and liposomes led to a multifaceted cytokine production in restimulated cells from spleen

CD4+ T cells play critical roles in mediating adaptive immunity to a variety of pathogens [64]. The predominance of Th1 and Th2 T helper cell subsets is known to result in distinct patterns of cytokine secretion. TLR ligands and also cationic liposomes typically increase Th1-biased CD4+ T-cell responses 
[34, 65], which have been associated with the protection conferred by different vaccines [64]. Therefore, the effect of GBS67-CpGODN adsorbed on DSPC: Cholesterol: DDA on the Th1 responses was further investigated.

After the last immunisation, splenocytes were isolated from all the mice and stimulated with GBS67 antigen $(4 \mu \mathrm{g} / \mathrm{mL})$ and RPMI as negative control. The profiles for 13 different cytokines were obtained by a cytometric bead array designed to phenotype mouse Th responses. This method was chosen as it allows simultaneous measurement of 13 cytokines efficiently in the large number of samples to obtain statistically reliable results.

Cytokine responses analysis revealed overall no significant differences between cytokines levels elicited by GBS67-CpGODN conjugate and GBS67, CpGODN mixture, presumably due to the low antigen and CPGODN doses used for the immunisation, which might not be sufficient for detectable T-cell proliferation induction. Similarly, the mixture of liposomes with CpGODN and GBS67 was not able to stimulate higher $\mathrm{T}$ cell mediated responses compared to the protein alone. In contrast, combination of cationic liposomes with protein conjugate demonstrated to trigger the highest cytokine production. Interestingly, a combination of cytokines released by Th1, Th2, Th17 and Th9 cells were noted, indicating a multifunctional activity of the protein conjugate-liposomes system, in line with the observed increased levels of both IgG1 and IgG2a (Fig. 7). Whether these differences in cytokine levels reflect differences differential expansion of T cell subsets although it remains possible that the observed difference are due to difference in cytokine production by similar numbers of T cells. This could be further investigated using a combination of intracellular staining for cytokines and surface staining with flow cytometry. However, irrespective of this, the results further corroborate that in order to achieve cell-mediated responses with such a low antigen and CpGODN dose, the covalent linkage of the CPGODN immunopotentiator to the protein prior to adsorption on particles surface, was needed. This effect is not biased towards a specific Th1 or Th2 response.
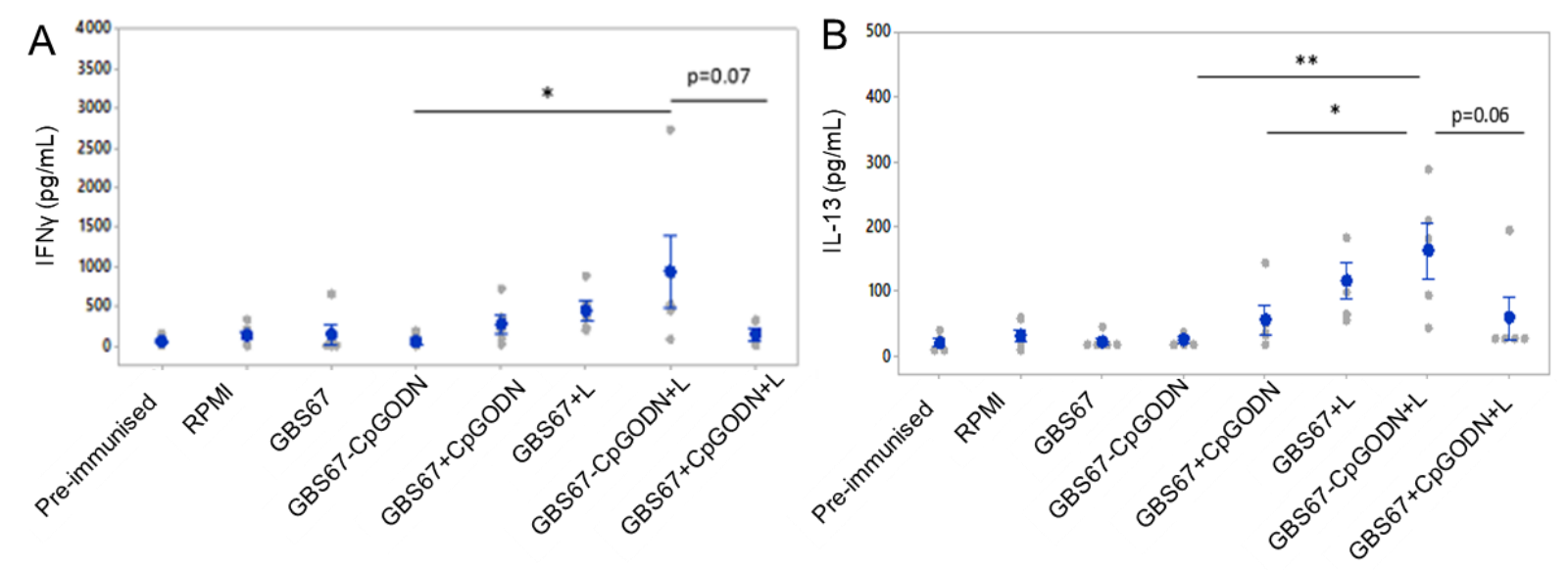

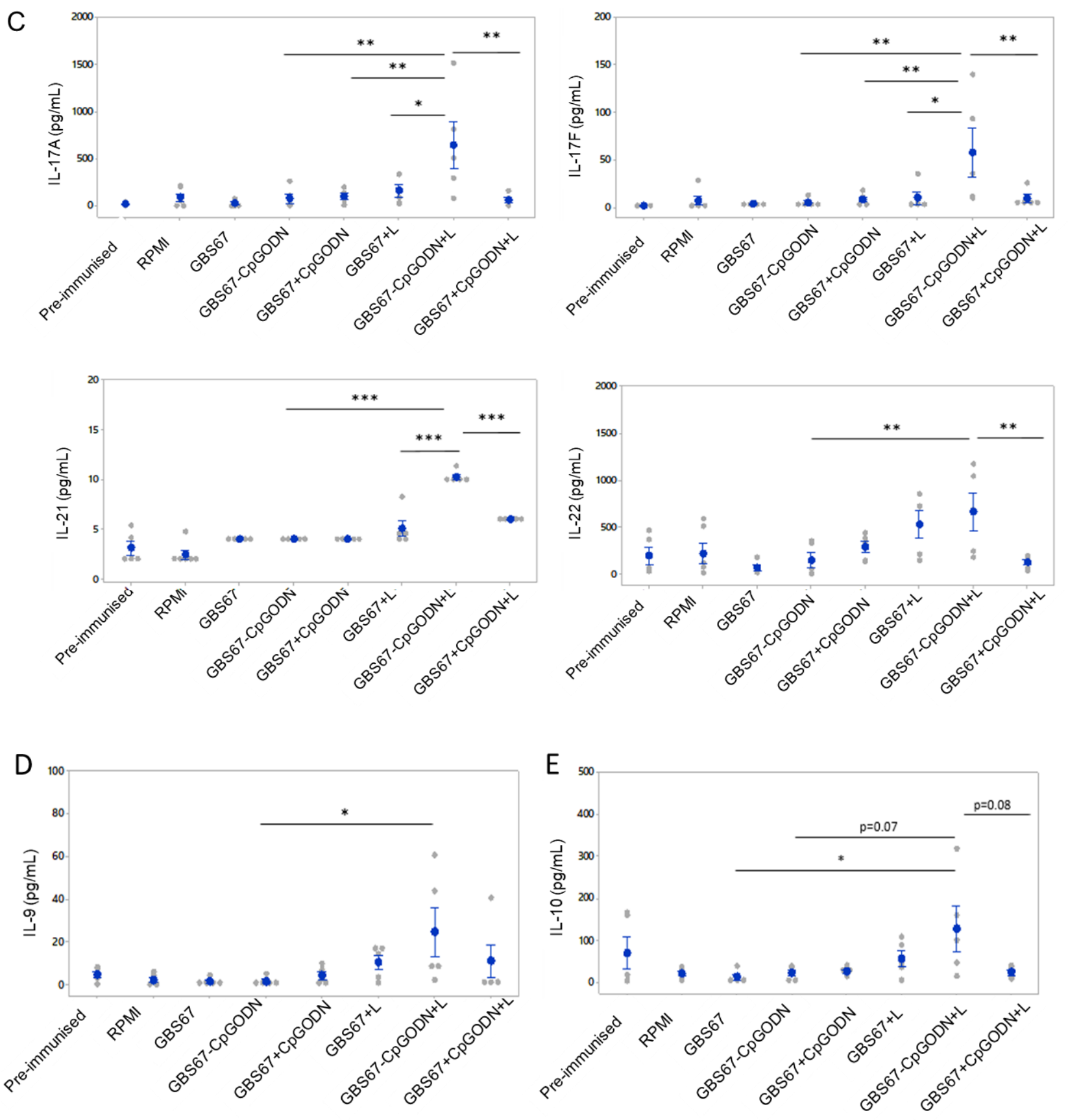

Fig. 7. Cytokine panels for (A) Th1 (B) Th2 (C) Th17 (D) Th9 and (E) Th cell-mediated responses. Mice were immunised with two injections spaced at 3-week intervals with the different formulations, as described in Section 2.10. At day 42, spleen cells were prepared and restimulated in vitro with $4 \mu \mathrm{g} / \mathrm{mL} \mathrm{GBS67}$ and were incubated at $37^{\circ} \mathrm{C}, 5 \% \mathrm{CO}_{2}$ for $72 \mathrm{hours}$. $\mathrm{RPMI}$ media was used as negative control. Culture supernatants were harvested after 72 hours and tested for cytokine levels by LEGENDplex mouse Th cytokine (13-plex) multi-analyte flow assay kit. Mixture and conjugate are represented by (+) and (-), respectively. Values represent the mean \pm SE from cells of five mice. ${ }^{*} p<0.05 ;{ }^{* *} p<0.01 ;{ }^{* *} p<0.001 ;$ ns: non-significant.

3.8 Adsorption of GBS67-CpGODN on cationic liposome surfaces prevents rapid biodistribution and promotes a depot of both liposomes and antigen at the injection site

525 Previous studies have suggested that the ability to form a depot at the injection site is important for 526 the function of many adjuvants and the depot forming effect of DDA-based liposomes has been demonstrated through a range of studies $[48,50,66]$. Based on this, the biodistribution profile of free 
528 GBS67, GBS67-CpGODN conjugate and GBS67-CpGODN adsorbed on DSPC: Cholesterol: DDA 529 liposomes, was compared to consider the ability of the liposome formulation to retain the antigen530 TLR agonist complex with the liposomes.

531 Overall, full body images showed that the signals of both antigens and liposomes are concentrated at 532 the site of injection for all the groups tested. After intramuscular injection of GBS67 either alone or 533 conjugated with CpGODN, rapid reduction of protein signal was noted as the study proceeded (Fig. 534 8A). Interestingly, part of the free protein and GBS67-conjugate dose was accumulated at the site of 535 injection for 11 days ( $14 \%$ and $21 \%$ of the initial dose signal remaining at day 11 post injection for 536 GBS67 and GBS67-CpGODN, respectively; Fig. 8B). Regarding GBS67-CpGODN, incorporation of 537 CPGODN TLR9 agonist in conjugated form, had no significant effect on protein retention at the 538 injection site, at all-time points measured (Fig. 8). Comparable levels of GBS67 remained at the 539 injection site (75\% remaining at day 1, $44 \%$ after 4 days, and $21 \%$ of the initial dose signal remaining 540 at day 11 post injection) when TLR9 agonist CpGODN was conjugated to GBS67 protein, although a 541 slower signal reduction was noted during the first 4 days of the study (Fig. 8). When considering 542 GBS67-CpGODN+liposomes retention at the site of injection, high levels of GBS67-CpGODN (114\%, $543128 \%$ and $116 \%$ of the antigen dose at days 1,7 and 11 post injection, respectively; Fig. $8 \mathrm{~A}$ and B) 544 were retained at the injection site, which were 8-fold and 5-fold higher than those obtained with free 545 GBS67 and GBS67-CpGODN protein conjugate at day $11(p<0.001)$. Interestingly, no DiD fluorescence 546 signal was detected immediately after injection. However, the liposome signal increased as the study 547 progressed with the final value at day 11 being 2 -fold higher than that obtained at day 1 (Fig. $8 \mathrm{C}$ ). This 548 may due to self-quenching of DiD occurring as a result of the initial aggregation of the cationic 549 liposomes due to their interaction with proteins present within the local biological milieu. This 550 aggregation results in the formation of a depot at the site of injection. The formation of a depot effect 551 after intramuscular injection has been previously reported with cationic liposomes e.g. [41, 47, 66]. 552 Within these studies, radio-labelled liposomes and antigens were tracked and shown to show strong 553 retention of the liposomes and their complexed protein antigen at the injection for up to 14 days, 554 similar to findings in Figure 8. 
A

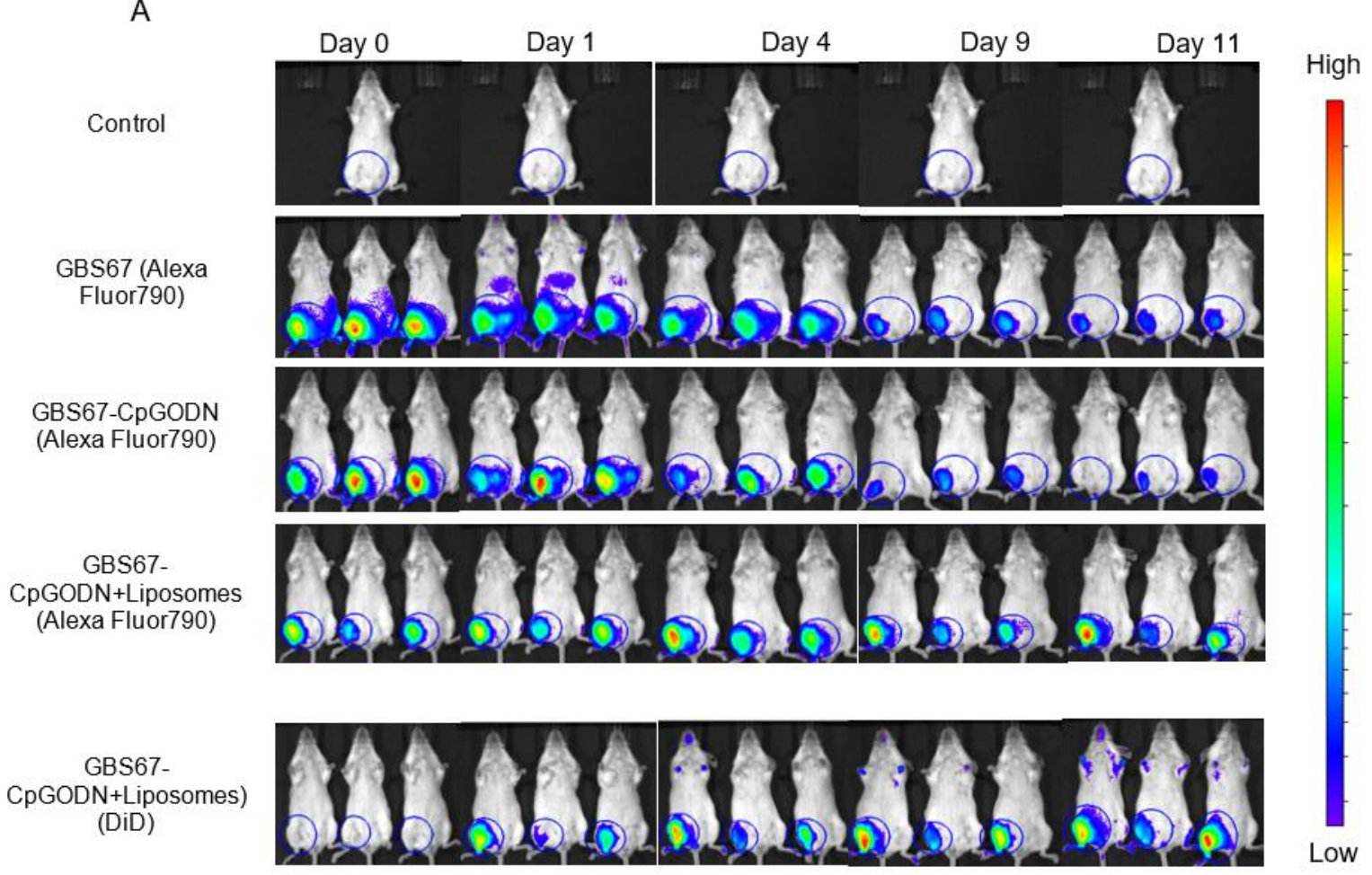

B

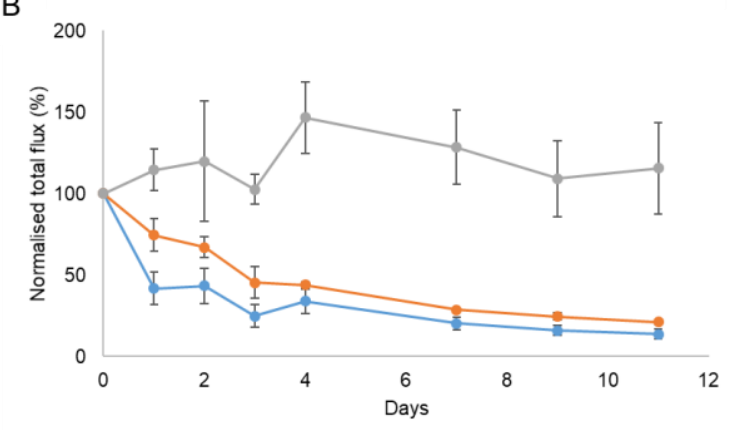

$\rightarrow$ GBS67 $\multimap-$ GBS67-CpGODN $\rightarrow-G B S 67-C p G O D N+L i p o s o m e s$
C

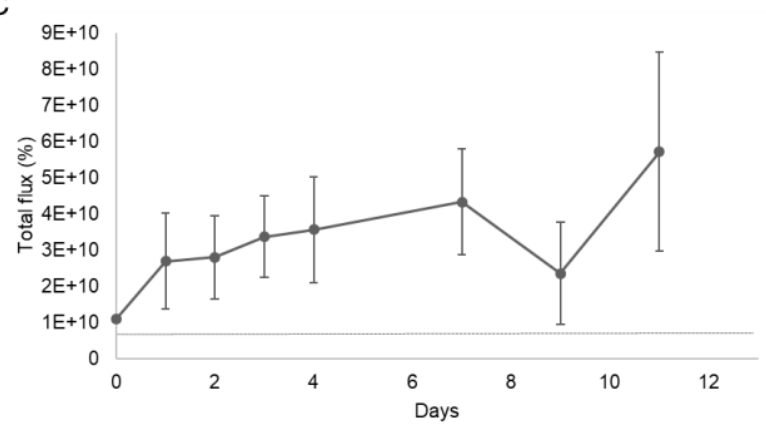

$\rightarrow$ GBS67-CpGODN+Liposomes

Fig. 8. Biodistribution profile of protein and liposomes. (A) Whole-body fluorescence intensity images for all groups for selected days. (B) Protein and (C) liposomes dose retention at the site of injection following intramuscular. injection of either free GBS67, GBS67 conjugated to CpGODN (GBS67-CpGODN) or GBS67-CpGODN adsorbed on the surface of DSPC: Cholesterol: DDA cationic liposomes (GBS67-CpGODN+Liposomes). Mice received $10 \mu \mathrm{g}$ protein-based dose of vaccine, corresponding to the administration of $1.5 \mu \mathrm{g}$ of TLR9 agonist. A $200 \mu \mathrm{g}$ dose of cationic liposomes was used in one of the groups. A naive mouse was used as negative control. The proportion of Alexa Fluor 790 tracking dye at the injection site as a percentage of the initial dose was calculated. Mixture and conjugation are represented by (+) and (-), respectively. Dash line on Figure (C) represents the background level. Results represent the mean \pm SD of three mice per group. ${ }^{* * *} p<0.001$.

\section{4. Conclusions}

The potency of CpG-like TLR9 agonists as vaccine adjuvants has been demonstrated by many preclinical studies and in clinical trials $[3,4,67,68]$. CpGODN has been used for stimulation of immune responses physically mixed with antigens and other adjuvants or encapsulated into nanoparticles for delivery to lymph nodes in an effort to protect it from degradation $[1,17]$. Particularly cationic liposomes have been demonstrated to induce a stronger immunogenicity than that of neutral and 
571 anionic liposomes [49, 69, 70], for their capability to adsorb negatively charged antigens, thus 572 improving the antigen presentation to APCs and providing a depot effect at the site of injection [71].

573 Furthermore, liposomes size seems optimal to increase the vaccine potency, as it is well established 574 that nanoparticles in the range of 20-200 nm favor transport to the draining lymph nodes [65].

575 Conjugation of $\mathrm{CpG}$ to protein antigens has been proven to enhance its adjuvant effect compared to 576 co-administration of antigen and adjuvant [15], by ensuring co-delivery of protein and adjuvant to the 577 same cell [3]. Similarly, cationic liposomes have attracted interest the last years due to their high 578 potential and efficiency as adjuvants/delivery systems for vaccine antigens $[48,65]$. Special focus has 579 been given on the use of cationic liposomes as their positive charge favours formation of the depot 580 effect at the injection site [72] followed by a sustained release to the draining lymph nodes [73]. 581 Building on this evidence, this work aimed at studying the liposomal delivery of CpGODN conjugated 582 on antigenic proteins in an effort to maximise its adjuvant potency.

583 Herein, GBS67 antigenic protein was successfully conjugated on CpGODN motifs using thiol-maleimide 584 chemistry $[43,45]$. Based on the isoelectric point of the conjugate and its negative charge, cationic 585 liposomes with DSPC: Cholesterol: DDA 10:40:50 composition and average size of 180-200 nm, 586 demonstrated the capability to adsorb on their surface the negatively charged adjuvant-protein 587 conjugate molecules to a very high degree. Immunological evaluation in mice evidenced that 588 presentation of protein conjugate on the surface of cationic nanoparticles elicited faster and enhanced 589 humoral responses compared to the adjuvant-protein conjugate as well as the protein mixed with the 590 adjuvant or liposome. The increase of total antibody IgG titres and cell-mediated immune responses 591 induced by the novel formulation also resulted in a higher bacterial opsonophagocytic killing in vitro. 592 Particularly, although the novel delivery system combining CpG conjugation and liposomes resulted in 593 a more pronounced production of the IgG2a subtype, respect to the mixture of $\mathrm{CpG}$ and liposomes, 594 and this could play a role in the highest antibody functionality [74], IgG1 subtypes were also 595 augmented. Interestingly, while CpG is known to induce a Th-1 biased response [2], the immune 596 response of our new formulation did not show a specific Th1/Th2-driven response. Finally, the 597 biodistribution study revealed high accumulation of protein conjugate+liposomes complex on the site 598 of injection compared to the protein conjugate alone, highlighting the benefit of liposomes 599 incorporation for a depot effect. Deposition of the vaccine formulation at the site of injection has been 600 proven to be an important factor for immunostimulatory action of vaccine as increases antigen 601 exposure time within the body [48].

602 Overall, a double adjuvanted system composed by CpGODN, antigen and cationic liposomes was 603 designed. The synergy of adjuvant-antigen conjugation with the liposome contribution described in 
604

605

606

607

608

609

610

611

612

613

614

615

616

617

618

619

620

621

622

623

624

625

626

627

628

629

630

631

632

633

634

635

636

637

638

639

640

641

this study can be particularly helpful for enhancement of immunity using low doses of antigen, increasing the speed of antigen-specific immune response generation and reducing the number of immunisations required to achieve effectiveness.

\section{Author contributions}

DC, STS, FC, MRR, CWR, YP and RA were involved in the conception and design of the study. DC, STS, IP, GB, FC, SW and RC acquired the data. DC, STS, RC, SW, CWR, YP and RA analysed and interpreted the results. DC, YP and RA prepared the manuscript. All authors were involved in drafting the manuscript or revising it critically for important intellectual content. All authors had full access to the data and approved the manuscript before it was submitted by the corresponding author.

\section{Conflict of interest}

GB, IP, FC, MRR, DO, UD and RA are employees of the GSK group of companies. RA and DO are owners of GSK stocks. Other authors declare no conflict of interest.

\section{Acknowledgments}

This work was funded by the European Commission Project Leveraging Pharmaceutical Sciences and Structural Biology Training to Develop $21^{\text {st }}$ Century Vaccines (H2020-MSCA-ITN-2015 Grant Agreement 675370) and Independent Research Fund Denmark (7026-00027B) (STS). All data included in this publication are openly available from the University of Strathclyde Knowledge Base at https://doi.org/10.15129/152a75e6-79d6-4b79-9e0c-d1ead37e894a.

\section{References}

1. Tam, Y.K., Liposomal encapsulation enhances the activity of immunostimulatory oligonucleotides. Future Lipidology, 2006. 1(1): p. 35-46.

2. de Titta, A., et al., Nanoparticle conjugation of CpG enhances adjuvancy for cellular immunity and memory recall at low dose. Proc Natl Acad Sci U S A, 2013. 110(49): p. 19902-7.

3. Vollmer, J. and A.M. Krieg, Immunotherapeutic applications of CpG oligodeoxynucleotide TLR9 agonists. Adv Drug Deliv Rev, 2009. 61(3): p. 195-204.

4. Scheiermann, J. and D.M. Klinman, Clinical evaluation of CpG oligonucleotides as adjuvants for vaccines targeting infectious diseases and cancer. Vaccine, 2014. 32(48): p. 6377-6389.

5. von Beust, B.R., et al., Improving the therapeutic index of CpG oligodeoxynucleotides by intralymphatic administration. Eur J Immunol, 2005. 35(6): p. 1869-76.

6. Goldinger, S.M., et al., Nano-particle vaccination combined with TLR-7 and -9 ligands triggers memory and effector CD8+ T-cell responses in melanoma patients. European Journal of Immunology, 2012. 42(11): p. 3049-3061.

7. Sparwasser, T., et al., Immunostimulatory CpG-Oligodeoxynucleotides Cause Extramedullary Murine Hemopoiesis. The Journal of Immunology, 1999. 162(4): p. 2368-2374.

8. Levin, A.A., A review of issues in the pharmacokinetics and toxicology of phosphorothioate antisense oligonucleotides. Biochimica et Biophysica Acta (BBA) - Gene Structure and Expression, 1999. 1489(1): p. 69-84. 
9. Henry, S.P., et al., Complement activation is responsible for acute toxicities in rhesus monkeys treated with a phosphorothioate oligodeoxynucleotide. International Immunopharmacology, 2002. 2(12): p. 1657-1666.

10. Scheiermann, J. and D.M. Klinman, Clinical evaluation of $C p G$ oligonucleotides as adjuvants for vaccines targeting infectious diseases and cancer. Vaccine, 2014. 32(48): p. 6377-89.

11. Hanagata, N., Structure-dependent immunostimulatory effect of CpG oligodeoxynucleotides and their delivery system. Int J Nanomedicine, 2012. 7: p. 2181-95.

12. Karbach, J., et al., Efficient <em>In vivo</em> Priming by Vaccination with Recombinant NYESO-1 Protein and CpG in Antigen Naïve Prostate Cancer Patients. Clinical Cancer Research, 2011. 17(4): p. 861-870.

13. Datta, S.K., et al., Antigen-immunostimulatory oligonucleotide conjugates: mechanisms and applications. Immunological Reviews, 2004. 199(1): p. 217-226.

14. Tighe, $\mathrm{H}$., et al., Conjugation of immunostimulatory DNA to the short ragweed allergen amb a 1 enhances its immunogenicity and reduces its allergenicity. J Allergy Clin Immunol, 2000. 106(1 Pt 1): p. 124-34.

15. Heit, A., et al., Protective CD8 T cell immunity triggered by CpG-protein conjugates competes with the efficacy of live vaccines. J Immunol, 2005. 174(7): p. 4373-80.

16. Kramer, K., et al., Intracellular Cleavable CpG Oligodeoxynucleotide-Antigen Conjugate Enhances Anti-tumor Immunity. Mol Ther, 2017. 25(1): p. 62-70.

17. Wilson, K.D., S.D. de Jong, and Y.K. Tam, Lipid-based delivery of C $p$ G oligonucleotides enhances immunotherapeutic efficacy. Adv Drug Deliv Rev, 2009. 61(3): p. 233-42.

18. Kuramoto, Y., et al., Efficient peritoneal dissemination treatment obtained by an immunostimulatory phosphorothioate-type CpG DNA/cationic liposome complex in mice. J Control Release, 2008. 126(3): p. 274-80.

19. Erikci, E., M. Gursel, and I. Gursel, Differential immune activation following encapsulation of immunostimulatory CpG oligodeoxynucleotide in nanoliposomes. Biomaterials, 2011. 32(6): p. 1715-23.

20. Bayyurt, B., et al., Encapsulation of two different TLR ligands into liposomes confer protective immunity and prevent tumor development. J Control Release, 2017. 247: p. 134-144.

21. Nikoofal-Sahlabadi, S., et al., Liposomal CpG-ODN: An in vitro and in vivo study on macrophage subtypes responses, biodistribution and subsequent therapeutic efficacy in mice models of cancers. Eur J Pharm Sci, 2018. 119: p. 159-170.

22. Rosini, R., et al., Identification of novel genomic islands coding for antigenic pilus-like structures in Streptococcus agalactiae. Molecular Microbiology, 2006. 61(1): p. 126-141.

23. Nobbs, A.H., et al., Sortase $A$ utilizes an ancillary protein anchor for efficient cell wall anchoring of pili in Streptococcus agalactiae. Infection and immunity, 2008. 76(8): p. 3550-3560.

24. Sharma, P., et al., Role of pilus proteins in adherence and invasion of Streptococcus agalactiae to the lung and cervical epithelial cells. J Biol Chem, 2013. 288(6): p. 4023-34.

25. Nilo, A., et al., Exploring the Effect of Conjugation Site and Chemistry on the Immunogenicity of an anti-Group B Streptococcus Glycoconjugate Vaccine Based on GBS67 Pilus Protein and Type V Polysaccharide. Bioconjugate Chemistry, 2015. 26(8): p. 1839-1849.

26. Campbell, J.R., et al., Group B streptococcal colonization and serotype-specific immunity in pregnant women at delivery. Obstetrics \& Gynecology, 2000. 96(4): p. 498-503.

27. Shabayek, S. and B. Spellerberg, Group B Streptococcal Colonization, Molecular Characteristics, and Epidemiology. Frontiers in Microbiology, 2018. 9(437).

28. Seale, A.C., et al., Estimates of the Burden of Group B Streptococcal Disease Worldwide for Pregnant Women, Stillbirths, and Children. Clinical Infectious Diseases, 2017. 65(suppl_2): p. S200-S219.

29. Nuccitelli, A., C.D. Rinaudo, and D. Maione, Group B Streptococcus vaccine: state of the art. Therapeutic advances in vaccines, 2015. 3(3): p. 76-90. 
30. Gizachew, M., et al., Streptococcus agalactiae maternal colonization, antibiotic resistance and serotype profiles in Africa: a meta-analysis. Annals of Clinical Microbiology and Antimicrobials, 2019. 18(1): p. 14.

31. Africa, C.W.J. and E. Kaambo, Group B Streptococcus Serotypes in Pregnant Women From the Western Cape Region of South Africa. Frontiers in public health, 2018. 6: p. 356-356.

32. Chohan, L., et al., Patterns of antibiotic resistance among group B streptococcus isolates: 20012004. Infectious diseases in obstetrics and gynecology, 2006. 2006: p. 57492-57492.

33. Margarit, I., et al., Preventing Bacterial Infections with Pilus-Based Vaccines: the Group B Streptococcus Paradigm. The Journal of Infectious Diseases, 2009. 199(1): p. 108-115.

34. Donadei, A., et al., The adjuvant effect of TLR7 agonist conjugated to a meningococcal serogroup C glycoconjugate vaccine. Eur J Pharm Biopharm, 2016. 107: p. 110-9.

35. Kastner, E., et al., High-throughput manufacturing of size-tuned liposomes by a new microfluidics method using enhanced statistical tools for characterization. International Journal of Pharmaceutics, 2014. 477(1): p. 361-368.

36. Joshi, S., et al., Microfluidics based manufacture of liposomes simultaneously entrapping hydrophilic and lipophilic drugs. International Journal of Pharmaceutics, 2016. 514(1): p. 160168.

37. Guimarães Sá Correia, M., et al., Microfluidic manufacturing of phospholipid nanoparticles: Stability, encapsulation efficacy, and drug release. International Journal of Pharmaceutics, 2017. 516(1): p. 91-99.

38. Hamborg, M., et al., Protein antigen adsorption to the DDA/TDB liposomal adjuvant: effect on protein structure, stability, and liposome physicochemical characteristics. Pharm Res, 2013. 30(1): p. 140-55.

39. Sangra, M., et al., Evidence of Protein Adsorption in Pegylated Liposomes: Influence of Liposomal Decoration. Nanomaterials (Basel), 2017. 7(2).

40. Forbes, N., et al., Rapid and scale-independent microfluidic manufacture of liposomes entrapping protein incorporating in-line purification and at-line size monitoring. International Journal of Pharmaceutics, 2019. 556: p. 68-81.

41. Lou, G., et al., A novel microfluidic-based approach to formulate size-tuneable large unilamellar cationic liposomes: Formulation, cellular uptake and biodistribution investigations. European Journal of Pharmaceutics and Biopharmaceutics, 2019. 143: p. 51-60.

42. Maurer, N., et al., Spontaneous Entrapment of Polynucleotides upon Electrostatic Interaction with Ethanol-Destabilized Cationic Liposomes. Biophysical Journal, 2001. 80(5): p. 2310-2326.

43. Clauson, R.M., B. Berg, and B. Chertok, The Content of CpG-DNA in Antigen-CpG Conjugate Vaccines Determines Their Cross-Presentation Activity. Bioconjug Chem, 2019. 30(3): p. 561567.

44. Jones, D.S., et al., A Method for Producing Protein Nanoparticles with Applications in Vaccines. PloS one, 2016. 11(3): p. e0138761-e0138761.

45. Maurer, T., et al., CpG-DNA aided cross-presentation of soluble antigens by dendritic cells. Eur J Immunol, 2002. 32(8): p. 2356-64.

46. Heit, A., et al., Cutting edge: Toll-like receptor 9 expression is not required for CpG DNA-aided cross-presentation of DNA-conjugated antigens but essential for cross-priming of CD8 T cells. J Immunol, 2003. 170(6): p. 2802-5.

47. Henriksen-Lacey, M., et al., Liposomes based on dimethyldioctadecylammonium promote a depot effect and enhance immunogenicity of soluble antigen. J Control Release, 2010. 142(2): p. 180-6.

48. Christensen, D., et al., Cationic liposomes as vaccine adjuvants. Expert Review of Vaccines, 2011. 10(4): p. 513-521.

49. Davidsen, J., et al., Characterization of cationic liposomes based on dimethyldioctadecylammonium and synthetic cord factor from M. tuberculosis (trehalose 6, 6'- 
dibehenate)-A novel adjuvant inducing both strong $C M I$ and antibody responses. Biochimica et Biophysica Acta (BBA) - Biomembranes, 2005. 1718(1): p. 22-31.

50. Henriksen-Lacey, M., et al., Comparison of the Depot Effect and Immunogenicity of Liposomes Based on Dimethyldioctadecylammonium (DDA), 36- $\left[N-\left(N^{\prime}, N^{\prime}-\right.\right.$ Dimethylaminoethane)carbomyl] Cholesterol (DC-Chol), and 1,2-Dioleoyl-3trimethylammonium Propane (DOTAP): Prolonged Liposome Retention Mediates Stronger Th1 Responses. Molecular Pharmaceutics, 2011. 8(1): p. 153-161.

51. van Dissel, J.T., et al., A novel liposomal adjuvant system, CAF01, promotes long-lived Mycobacterium tuberculosis-specific T-cell responses in human. Vaccine, 2014. 32(52): p. 7098-7107.

52. Gao, X. and G.V. Lowry, Progress towards standardized and validated characterizations for measuring physicochemical properties of manufactured nanomaterials relevant to nano health and safety risks. Nanolmpact, 2018. 9: p. 14-30.

53. Caputo, F., et al., Measuring particle size distribution of nanoparticle enabled medicinal products, the joint view of EUNCL and NCI-NCL. A step by step approach combining orthogonal measurements with increasing complexity. J Control Release, 2019. 299: p. 31-43.

54. Maguire, C.M., et al., Characterisation of particles in solution - a perspective on light scattering and comparative technologies. Sci Technol Adv Mater, 2018. 19(1): p. 732-745.

55. Kovacs-Nolan, J., et al., The novel adjuvant combination of CpG ODN, indolicidin and polyphosphazene induces potent antibody-and cell-mediated immune responses in mice. Vaccine, 2009. 27(14): p. 2055-64.

56. Zhao, B.G., et al., Combination therapy targeting toll like receptors 7, 8 and 9 eliminates large established tumors. Journal for ImmunoTherapy of Cancer, 2014. 2(1): p. 12.

57. Moyer, T.J., A.C. Zmolek, and D.J. Irvine, Beyond antigens and adjuvants: formulating future vaccines. J Clin Invest, 2016. 126(3): p. 799-808.

58. Brigtsen, A.K., et al., Induction of Cross-Reactive Antibodies by Immunization of Healthy Adults with Types la and Ib Group B Streptococcal Polysaccharide-Tetanus Toxoid Conjugate Vaccines. The Journal of Infectious Diseases, 2002. 185(9): p. 1277-1284.

59. Guttormsen, H.-K., Y. Liu, and L.C. Paoletti, Functional activity of antisera to group B streptococcal conjugate vaccines measured with an opsonophagocytosis assay and HL-60 effector cells. Human vaccines, 2008. 4(5): p. 370-374.

60. Shirota, H., et al., Novel roles of CpG oligodeoxynucleotides as a leader for the sampling and presentation of CpG-tagged antigen by dendritic cells. J Immunol, 2001. 167(1): p. 66-74.

61. Tao, Y., et al., Engineered CpG-Antigen Conjugates Protected Gold Nanoclusters as Smart SelfVaccines for Enhanced Immune Response and Cell Imaging. Advanced Functional Materials, 2014. 24(7): p. 1004-1010.

62. Hanagata, N., CPG oligodeoxynucleotide nanomedicines for the prophylaxis or treatment of cancers, infectious diseases, and allergies. International Journal of Nanomedicine, 2017. Volume 12: p. 515-531.

63. Ignacio, B.J., et al., Toll-like Receptor Agonist Conjugation: A Chemical Perspective. Bioconjug Chem, 2018. 29(3): p. 587-603.

64. Zhu, J., H. Yamane, and W.E. Paul, Differentiation of effector CD4 T cell populations (*). Annu Rev Immunol, 2010. 28: p. 445-89.

65. Perrie, Y., et al., Designing liposomal adjuvants for the next generation of vaccines. Adv Drug Deliv Rev, 2016. 99(Pt A): p. 85-96.

66. Wilkinson, A., et al., Lipid conjugation of TLR7 agonist Resiquimod ensures co-delivery with the liposomal Cationic Adjuvant Formulation 01 (CAFO1) but does not enhance immunopotentiation compared to non-conjugated Resiquimod+CAF01. J Control Release, 2018. 291: p. 1-10.

67. Shi, S., et al., Vaccine adjuvants: Understanding the structure and mechanism of adjuvanticity. Vaccine, 2019. 37(24): p. 3167-3178. 
68. Janssen, R.S., et al., Immunogenicity and safety of an investigational hepatitis $B$ vaccine with a toll-like receptor 9 agonist adjuvant (HBsAg-1018) compared with a licensed hepatitis $B$ vaccine in patients with chronic kidney disease. Vaccine, 2013. 31(46): p. 5306-13.

69. Yan, W., W. Chen, and L. Huang, Mechanism of adjuvant activity of cationic liposome: Phosphorylation of a MAP kinase, ERK and induction of chemokines. Molecular Immunology, 2007. 44(15): p. 3672-3681.

70. Vangala, A., et al., A comparative study of cationic liposome and niosome-based adjuvant systems for protein subunit vaccines: characterisation, environmental scanning electron microscopy and immunisation studies in mice. Journal of Pharmacy and Pharmacology, 2006. 58(6): p. 787-799.

71. Henriksen-Lacey, M., et al., Liposomal cationic charge and antigen adsorption are important properties for the efficient deposition of antigen at the injection site and ability of the vaccine to induce a CMI response. J Control Release, 2010. 145(2): p. 102-8.

72. Roces, C.B., et al., Scale-Independent Microfluidic Production of Cationic Liposomal Adjuvants and Development of Enhanced Lymphatic Targeting Strategies. Mol Pharm, 2019. 16(10): p. 4372-4386.

73. Awate, S., L.A. Babiuk, and G. Mutwiri, Mechanisms of action of adjuvants. Front Immunol, 2013. 4: p. 114.

74. Vidarsson, G., G. Dekkers, and T. Rispens, IgG subclasses and allotypes: from structure to effector functions. Frontiers in immunology, 2014. 5: p. 520-520. 